Supplement of Earth Surf. Dynam., 8, 303-322, 2020

https://doi.org/10.5194/esurf-8-303-2020-supplement

(C) Author(s) 2020. This work is distributed under

the Creative Commons Attribution 4.0 License.

(c) (i)
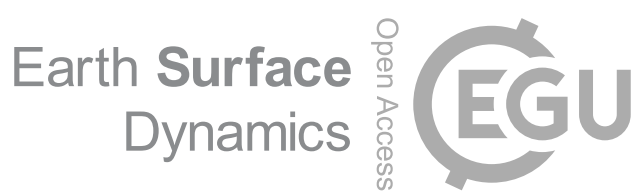

Supplement of

\title{
Interactions between main channels and tributary alluvial fans: channel adjustments and sediment-signal propagation
}

Sara Savi et al.

Correspondence to: Sara Savi (savi@geo.uni-potsdam.de)

The copyright of individual parts of the supplement might differ from the CC BY 4.0 License. 


\section{Supplementary material}

This document contains the measured and calculated data used for the analyses reported in the main manuscript. In the following pages the reader finds the raw data of the slope and $Q_{s_{-} \text {out }}$ measurements (Table S1 and S2) and of the volumes calculations (Tables S3 to S8). Additional data figures are also provided in support of the information reported in the main manuscript (Table S9 and Figure S1 to S9). 
Table S1. Manually measured main channel slope (\%) and Qs_out values ( $\mathrm{ml} / \mathrm{s}$; normalized to $Q_{\left.s_{-} i n\right)}$

\begin{tabular}{|c|c|c|c|c|c|c|c|c|c|c|c|c|}
\hline \multirow{2}{*}{$\frac{\text { Run time }}{(\text { min })}$} & \multicolumn{2}{|c|}{ MC_NC } & \multicolumn{2}{|c|}{ T_NC1 } & \multicolumn{2}{|c|}{ T_ISDS } & \multicolumn{2}{|c|}{ T_DWIW } & \multicolumn{2}{|c|}{ T_NC2 } & \multicolumn{2}{|c|}{ T_IWMC } \\
\hline & Slope & $Q_{s_{\text {_out }}}$ & Slope & $Q_{s_{\text {_out }}}$ & Slope & $Q_{s_{\text {out }}}$ & Slope & $Q_{s_{\text {oout }}}$ & Slope & $Q_{s_{\text {_out }}}$ & Slope & $Q_{\text {s_out }}$ \\
\hline 0 & 5.3 & & 4.5 & & 4.8 & & 5.0 & & 4.6 & & 5.3 & \\
\hline 10 & 7.3 & 21.3 & 6.8 & 14.0 & 6.8 & 19.8 & 7.4 & 10.1 & 7.1 & 5.9 & 6.9 & 5.6 \\
\hline 20 & 7.2 & 16.7 & 7.0 & 6.9 & 6.8 & 12.4 & 7.6 & 13.4 & 7.3 & 5.9 & 7.0 & 7.2 \\
\hline 30 & 7.3 & 7.0 & 6.9 & 8.6 & 7.1 & 7.3 & 7.5 & 11.1 & 7.2 & 3.2 & 7.1 & 2.9 \\
\hline 40 & 7.4 & 9.7 & 7.0 & 4.6 & 6.9 & 4.2 & 7.9 & 7.9 & 7.3 & 3.6 & 7.4 & 3.9 \\
\hline 50 & 7.6 & 10.1 & 7.1 & 8.9 & 7.3 & 8.6 & 7.8 & 8.5 & 7.4 & 2.9 & 7.5 & 2.6 \\
\hline 60 & 7.5 & 9.8 & 7.3 & 7.2 & 7.0 & 7.9 & 7.8 & 9.2 & 7.4 & 4.0 & 7.5 & 1.8 \\
\hline 70 & 7.6 & 6.4 & 7.3 & 6.2 & 7.3 & 8.1 & 8.0 & 6.3 & 7.7 & 3.4 & 7.4 & 2.0 \\
\hline 80 & 7.7 & 9.1 & 7.4 & 4.6 & 7.4 & 4.9 & 8.0 & 4.7 & 7.9 & 2.3 & 7.6 & 2.0 \\
\hline 90 & 7.8 & 5.1 & 7.4 & & 7.5 & 4.3 & 8.0 & 3.6 & 7.8 & 3.0 & 7.7 & 2.4 \\
\hline 100 & 7.7 & 10.1 & 7.5 & 7.0 & 7.6 & 4.3 & 8.0 & 5.9 & 7.8 & 2.1 & 7.8 & 2.1 \\
\hline 110 & 7.6 & 4.3 & 7.6 & 2.6 & 7.6 & 4.6 & 8.1 & 2.3 & 7.9 & 4.9 & 7.9 & 1.6 \\
\hline 120 & 7.4 & 9.0 & 7.5 & 2.6 & 7.6 & 8.3 & 8.1 & 4.3 & 7.9 & 4.5 & 7.8 & 1.9 \\
\hline 130 & 7.6 & 3.6 & 7.4 & 9.8 & 7.9 & 3.4 & 8.1 & 3.3 & 8.1 & 4.2 & 7.9 & 4.3 \\
\hline 140 & 7.4 & 3.6 & 7.5 & 2.0 & 8.1 & 4.3 & 8.0 & 2.9 & 8.1 & 4.0 & 7.9 & 3.2 \\
\hline 150 & 7.4 & 4.3 & 7.6 & 5.0 & 7.9 & 2.3 & 7.9 & 3.4 & 8.2 & 1.4 & 8.0 & 2.4 \\
\hline 160 & 7.4 & 5.9 & 7.8 & 4.7 & 8.0 & 4.4 & 8.0 & 3.9 & 8.1 & 2.5 & 8.0 & 2.0 \\
\hline 170 & 7.6 & 8.2 & 7.8 & 2.7 & 7.9 & 2.9 & 8.0 & 4.3 & 8.3 & 2.3 & 8.2 & 1.3 \\
\hline 180 & 7.4 & 4.0 & 7.8 & & 7.9 & 4.6 & 8.0 & 3.2 & 8.2 & 2.9 & 8.1 & 2.7 \\
\hline 190 & 7.4 & 4.2 & 7.6 & 5.1 & 7.9 & 1.7 & 8.0 & 3.0 & 8.1 & 1.9 & 7.5 & 4.7 \\
\hline 200 & 7.5 & 3.9 & 7.5 & 4.3 & 7.9 & 3.7 & 8.0 & 2.2 & 8.3 & 3.2 & 7.4 & 3.9 \\
\hline 210 & 7.3 & 3.4 & 7.3 & 7.3 & 7.6 & 4.9 & 8.0 & 2.4 & 8.3 & 1.3 & 7.3 & 3.7 \\
\hline 220 & 7.2 & 2.9 & 7.6 & 3.2 & 7.6 & 2.9 & 8.0 & 3.6 & 8.2 & 2.9 & 7.3 & 5.7 \\
\hline 230 & 7.3 & 3.6 & 7.5 & 2.4 & 7.6 & 6.0 & 7.9 & 3.2 & 8.3 & 1.9 & 7.1 & 2.9 \\
\hline 240 & 7.1 & 2.9 & 7.6 & 4.1 & 7.6 & 5.7 & 7.6 & 2.8 & 8.3 & 2.6 & 6.9 & 6.4 \\
\hline 250 & 7.1 & 2.3 & 7.5 & 3.0 & 7.8 & 2.6 & 7.6 & 3.7 & 8.3 & 2.4 & 7.1 & 2.6 \\
\hline 260 & 7.1 & 3.6 & 7.6 & 6.3 & 7.5 & 3.3 & 7.8 & 3.2 & 8.4 & 1.9 & 7.0 & 2.5 \\
\hline 270 & 7.1 & 2.6 & 7.4 & 2.9 & 7.6 & 2.0 & 7.6 & 3.7 & 8.3 & 1.3 & 7.0 & 3.4 \\
\hline 280 & 7.2 & 5.6 & 7.5 & 2.9 & 7.8 & 2.6 & 7.6 & 3.2 & 8.4 & 1.7 & 7.1 & 3.2 \\
\hline 290 & 7.3 & 3.3 & 7.6 & 4.9 & 7.8 & 3.0 & 7.4 & 4.0 & 8.5 & 3.0 & 7.0 & 2.6 \\
\hline 300 & 7.0 & 5.4 & 7.5 & & 7.6 & 3.5 & 7.3 & 5.7 & 8.4 & 2.7 & 6.9 & 2.0 \\
\hline 310 & 7.1 & 2.8 & 7.5 & 2.9 & 7.4 & 1.2 & 7.4 & 1.8 & 8.4 & 1.3 & 6.9 & 3.7 \\
\hline 320 & 7.1 & 5.2 & 7.5 & 2.6 & 7.6 & 1.8 & 7.1 & 3.3 & 8.6 & 1.9 & 7.0 & 3.4 \\
\hline 330 & 7.0 & 4.0 & 7.5 & 2.6 & 7.6 & 2.2 & 7.0 & 1.6 & 8.5 & 0.9 & 6.8 & 2.3 \\
\hline 340 & 7.1 & 3.0 & 7.4 & 5.9 & 7.3 & 2.4 & 7.3 & 1.6 & 8.6 & 1.7 & 6.9 & 3.3 \\
\hline 350 & 7.1 & 4.4 & 7.4 & 3.6 & 7.3 & 1.5 & 7.1 & $\begin{array}{l}1.4 \\
\end{array}$ & 8.6 & 1.3 & 6.9 & 2.2 \\
\hline 360 & 7.1 & 3.6 & 7.4 & 3.9 & 7.4 & 2.8 & 7.2 & 1.9 & 8.5 & 2.7 & 6.8 & 2.2 \\
\hline 370 & 7.0 & 2.0 & 7.4 & 3.9 & 7.5 & 1.7 & 7.3 & 1.7 & 8.6 & 1.6 & 6.9 & 3.3 \\
\hline 380 & 7.1 & 3.1 & 7.1 & 2.5 & 7.5 & $\begin{array}{ll}1.1 \\
\end{array}$ & 7.3 & 1.7 & 8.6 & 0.9 & 6.9 & 2.8 \\
\hline 390 & 6.9 & 4.7 & 7.4 & 5.3 & 7.8 & 0.9 & 7.2 & 2.0 & 8.5 & 2.0 & 6.8 & 2.2 \\
\hline 400 & 7.1 & 2.3 & 7.3 & 1.7 & 7.6 & 1.2 & 7.4 & 3.7 & 8.6 & 2.2 & 6.8 & 2.7 \\
\hline 410 & 7.1 & 2.3 & 7.3 & 3.4 & 7.5 & 1.6 & 7.1 & 5.2 & 8.7 & 2.6 & 7.0 & 4.9 \\
\hline 420 & 7.0 & 3.2 & 7.4 & 2.0 & 7.4 & & 7.3 & 2.3 & 8.4 & 2.1 & 7.0 & 2.1 \\
\hline 430 & 6.9 & 2.8 & 7.1 & 1.8 & 7.3 & 1.2 & 7.3 & 3.4 & 8.6 & 2.3 & 7.0 & 3.4 \\
\hline 440 & 7.0 & 3.6 & 7.3 & 1.5 & 7.3 & 0.5 & 7.1 & 4.2 & 8.6 & 1.4 & 6.9 & 4.9 \\
\hline 450 & 6.9 & 4.2 & 7.3 & 1.5 & 7.3 & 2.4 & 7.3 & 3.2 & 8.6 & 1.0 & 6.9 & 2.3 \\
\hline 460 & 6.9 & 1.4 & 7.3 & 1.3 & 7.1 & 1.9 & 7.3 & 3.4 & 8.6 & 1.5 & 6.9 & 5.2 \\
\hline 470 & 7.1 & 2.8 & 7.1 & 1.7 & 7.1 & 2.3 & 7.5 & 2.6 & 8.5 & 2.7 & 6.6 & 2.5 \\
\hline 480 & 7.0 & 2.4 & 7.1 & 1.9 & 7.3 & 1.7 & 7.5 & 1.9 & 8.4 & 1.8 & 6.8 & 4.3 \\
\hline 490 & 7.0 & 1.9 & 7.3 & 1.9 & 7.4 & 2.5 & 7.3 & 3.9 & & & & \\
\hline 500 & 6.9 & 1.3 & 6.9 & 1.6 & 7.6 & 1.4 & 7.1 & 4.7 & & & & \\
\hline 510 & 6.9 & 3.1 & 6.8 & 1.6 & 7.4 & 1.7 & 7.2 & 2.5 & & & & \\
\hline 520 & 6.9 & 2.0 & 6.9 & 1.6 & 7.3 & 1.1 & 7.3 & 1.6 & & & & \\
\hline 530 & 6.9 & 1.7 & 6.8 & 1.4 & 7.3 & 4.5 & 7.4 & 2.2 & & & & \\
\hline
\end{tabular}




\begin{tabular}{|l|l|l|l|l|l|l|l|l|l|l|l|l|}
\hline 540 & 7.0 & 1.4 & 6.6 & 2.2 & 7.1 & 2.6 & 7.3 & 2.7 & & & & \\
\hline 550 & 6.8 & 2.0 & 6.8 & 1.7 & 7.1 & 1.1 & 7.2 & 3.0 & & & & \\
\hline 560 & 6.9 & 2.3 & 6.8 & 1.4 & 7.0 & 3.9 & 7.3 & 4.2 & & & & \\
\hline 570 & 6.9 & 2.9 & 6.5 & 1.3 & 7.1 & 6.3 & 7.4 & & & & & \\
\hline 580 & 7.0 & 2.1 & 6.5 & 1.8 & 7.1 & 2.1 & 7.3 & 2.2 & & & & \\
\hline 590 & 6.9 & 2.2 & 6.6 & 1.3 & 7.4 & 2.1 & 7.4 & 2.0 & & & & \\
\hline 600 & 6.8 & 1.9 & 6.5 & 0.0 & 7.3 & 2.6 & 7.3 & & & & & \\
\hline 610 & 7.0 & 4.7 & & & 7.3 & 2.6 & 7.3 & 2.1 & & & & \\
\hline 620 & 7.0 & 1.4 & & & 7.3 & 2.2 & 7.4 & 2.7 & & & & \\
\hline 630 & 6.8 & 1.6 & & & 7.3 & 2.4 & 7.4 & 2.3 & & & & \\
\hline 640 & 6.8 & 2.9 & & & 7.3 & 3.0 & 7.3 & 1.7 & & & & \\
\hline 650 & 6.9 & 2.3 & & & 7.1 & 1.4 & 7.3 & 1.8 & & & & \\
\hline 660 & 6.8 & 2.2 & & & 7.2 & 2.6 & 7.2 & 1.7 & & & & \\
\hline 670 & 7.1 & 1.9 & & & 7.3 & 0.9 & & & & & & \\
\hline 680 & 6.7 & 1.2 & & & 7.3 & 1.7 & & & & & & \\
\hline 690 & 6.7 & 2.5 & & & 7.1 & 2.4 & & & & & & \\
\hline 700 & & & & & 7.1 & 1.8 & & & & & & \\
\hline 710 & & & & & 7.1 & 1.4 & & & & & & \\
\hline 720 & & & & & 7.3 & 1.2 & & & & & & \\
\hline
\end{tabular}

Table S2. Calculated slope (\%) for the tributary channel (using DEMs).

\begin{tabular}{|r|r|r|r|r|r|}
\hline $\begin{array}{c}\text { Runtime } \\
\text { (min) }\end{array}$ & T_NC1 & T_ISDS & T_DWIW & T_NC2 & T_IWMC \\
\hline 30 & 11.0 & 11.2 & 9.4 & 12.8 & 10.6 \\
\hline 60 & 8.6 & 9.9 & 12.0 & 12.5 & 12.7 \\
\hline 90 & 9.6 & 11.7 & 10.9 & 12.6 & 12.3 \\
\hline 120 & 9.6 & 10.9 & 11.1 & 13.2 & 13.2 \\
\hline 150 & 9.7 & 11.7 & 10.5 & 11.7 & 12.4 \\
\hline 180 & 6.4 & 10.3 & 11.3 & 11.5 & 12.0 \\
\hline 210 & 8.2 & 9.0 & 10.7 & 11.1 & 11.9 \\
\hline 240 & 7.4 & 9.1 & 10.4 & 12.0 & 13.0 \\
\hline 270 & 10.0 & 8.0 & 11.0 & 10.9 & 13.5 \\
\hline 300 & 9.1 & 8.2 & 9.7 & 13.1 & 12.5 \\
\hline 330 & 11.4 & 8.3 & 11.5 & 10.3 & 12.2 \\
\hline 360 & 11.8 & 11.0 & 11.1 & 11.4 & 11.4 \\
\hline 390 & 10.5 & 11.4 & 13.0 & 11.4 & 11.7 \\
\hline 420 & 8.6 & 11.9 & 11.2 & 12.1 & 12.0 \\
\hline 450 & 9.3 & 11.9 & 10.4 & 10.2 & 13.2 \\
\hline 480 & 8.7 & 10.2 & 9.1 & 11.1 & 12.0 \\
\hline 510 & 8.8 & 12.6 & 10.4 & & \\
\hline 540 & 10.8 & 12.5 & 9.8 & & \\
\hline 570 & 8.9 & 9.4 & 9.2 & & \\
\hline 600 & 10.5 & 10.8 & 9.0 & & \\
\hline 630 & & 10.2 & 10.1 & & \\
\hline 660 & & 10.1 & 9.8 & & \\
\hline 690 & & 9.6 & 10.4 & & \\
\hline 720 & & 9.6 & & & \\
\hline & & & & & \\
\hline
\end{tabular}


Table S3 to S8. Volumes of mobilize sediment for each experiment. Data are divided into upper, middle, and lower sections. The volumes mobilized in the whole system is additionally provided at the bottom of each experiment table. The bank contribution in percent is calculated on the total volume of sediment exiting the system.

Table S3. MC_NC experiment. All values are reported in $10^{-3} \mathrm{~m}^{3}$ over a period of 30 minutes (Run time is in minutes).

\begin{tabular}{|c|c|c|c|c|c|c|}
\hline \multicolumn{7}{|c|}{ Upper section } \\
\hline $\begin{array}{l}\text { Run } \\
\text { time }\end{array}$ & $\begin{array}{r}\text { DE } \\
\mathbf{M}\end{array}$ & $\begin{array}{r}\text { Aggradation } \\
\left(A_{v f}\right)\end{array}$ & $\begin{array}{r}\text { Erosion } \\
\left(\boldsymbol{E}_{\boldsymbol{v} f f}\right)\end{array}$ & $\begin{array}{r}\text { Bank erosion } \\
\left(E_{b}\right)\end{array}$ & $\begin{array}{r}\text { Bank deposits } \\
\left(A_{b}\right)\end{array}$ & $\begin{array}{r}\text { Bank contribution } \\
\left(V_{b}\right)\end{array}$ \\
\hline 60 & 3 & 1.490 & -1.010 & 0.000 & 0.000 & 0.000 \\
\hline 90 & 4 & 0.422 & -1.841 & -0.491 & 0.000 & 0.491 \\
\hline 120 & 5 & 0.058 & -1.590 & -0.212 & 0.000 & 0.212 \\
\hline 150 & 6 & 0.173 & -1.986 & -0.024 & 0.000 & 0.024 \\
\hline 180 & 8 & 0.037 & -1.462 & 0.000 & 0.000 & 0.000 \\
\hline 210 & 9 & 0.118 & -0.843 & -0.001 & 0.000 & 0.001 \\
\hline 240 & 10 & 0.107 & -1.768 & 0.000 & 0.000 & 0.000 \\
\hline 270 & 11 & 0.416 & -1.054 & 0.000 & 0.000 & 0.000 \\
\hline 300 & 12 & 0.270 & -0.638 & 0.000 & 0.000 & 0.000 \\
\hline 330 & 13 & 0.473 & -1.032 & -0.018 & 0.000 & 0.018 \\
\hline 360 & 14 & 0.129 & -1.222 & 0.000 & 0.000 & 0.000 \\
\hline 390 & 15 & 0.092 & -1.064 & 0.000 & 0.000 & 0.000 \\
\hline 420 & 16 & 0.212 & -0.836 & -0.033 & 0.000 & 0.032 \\
\hline 450 & 17 & 0.455 & -0.710 & -0.126 & 0.001 & 0.125 \\
\hline 480 & 19 & 0.279 & -0.521 & -0.055 & 0.001 & 0.054 \\
\hline 510 & 20 & 0.110 & -0.558 & -0.160 & 0.002 & 0.158 \\
\hline 540 & 21 & 0.332 & -0.518 & -0.224 & 0.000 & 0.224 \\
\hline 570 & 22 & 0.096 & -0.727 & -0.313 & 0.000 & 0.313 \\
\hline 600 & 23 & 0.512 & -0.400 & -0.045 & 0.002 & 0.043 \\
\hline 630 & 24 & 0.433 & -0.429 & -0.124 & 0.085 & 0.039 \\
\hline 660 & 25 & 0.040 & -1.500 & -0.201 & 0.000 & 0.201 \\
\hline 690 & 26 & 0.168 & -0.680 & -0.006 & 0.001 & 0.005 \\
\hline \multicolumn{7}{|c|}{ Middle section } \\
\hline $\begin{array}{l}\text { Run } \\
\text { time }\end{array}$ & $\begin{array}{r}\mathbf{D E} \\
\mathbf{M} \\
\end{array}$ & $\begin{array}{r}\text { Aggradation } \\
\left(A_{v f}\right) \\
\end{array}$ & $\begin{array}{r}\text { Erosion } \\
\left(\boldsymbol{E}_{v f}\right) \\
\end{array}$ & $\begin{array}{r}\text { Bank erosion } \\
\left(E_{b}\right)\end{array}$ & $\begin{array}{r}\text { Bank deposits } \\
\left(A_{b}\right) \\
\end{array}$ & $\begin{array}{r}\text { Bank contribution } \\
\left(V_{b}\right) \\
\end{array}$ \\
\hline 60 & 3 & 0.002 & -3.665 & -2.831 & 0.000 & 2.831 \\
\hline 90 & 4 & 0.041 & -2.736 & -1.730 & 0.000 & 1.730 \\
\hline 120 & 5 & 0.367 & -0.927 & -2.467 & 0.000 & 2.467 \\
\hline 150 & 6 & 0.077 & -1.875 & -1.201 & 0.001 & 1.200 \\
\hline 180 & 8 & 0.257 & -1.394 & -0.560 & 0.002 & 0.558 \\
\hline 210 & 9 & 0.005 & -2.815 & -0.120 & 0.002 & 0.118 \\
\hline 240 & 10 & 0.982 & -0.729 & -1.804 & 0.011 & 1.793 \\
\hline 270 & 11 & 0.849 & -0.840 & -1.363 & 0.006 & 1.357 \\
\hline 300 & 12 & 0.125 & -1.919 & -1.342 & 0.109 & 1.233 \\
\hline 330 & 13 & 0.300 & -1.869 & -0.917 & 0.007 & 0.909 \\
\hline 360 & 14 & 0.151 & -1.641 & -0.418 & 0.094 & 0.324 \\
\hline 390 & 15 & 0.672 & -1.000 & -0.881 & 0.031 & 0.850 \\
\hline 420 & 16 & 0.391 & -1.166 & -0.419 & 0.001 & 0.418 \\
\hline 450 & 17 & 0.280 & -1.006 & -0.404 & 0.101 & 0.302 \\
\hline 480 & 19 & 0.650 & -0.504 & -0.157 & 0.029 & 0.128 \\
\hline 510 & 20 & 0.457 & -0.479 & -0.001 & 0.008 & -0.007 \\
\hline 540 & 21 & 0.442 & -0.649 & -0.533 & 0.004 & 0.529 \\
\hline 570 & 22 & 0.345 & -1.208 & -0.279 & 0.105 & 0.174 \\
\hline 600 & 23 & 0.963 & -0.955 & -0.958 & 0.001 & 0.957 \\
\hline 630 & 24 & 0.471 & -1.615 & -1.104 & 0.345 & 0.759 \\
\hline 660 & 25 & 0.964 & -0.947 & -0.222 & 0.078 & 0.144 \\
\hline 690 & 26 & 0.714 & -0.959 & -1.133 & 0.278 & 0.856 \\
\hline
\end{tabular}




\begin{tabular}{|c|c|c|c|c|c|c|}
\hline \multicolumn{7}{|c|}{ Lower section } \\
\hline $\begin{array}{l}\text { Run } \\
\text { time }\end{array}$ & $\begin{array}{r}\text { DE } \\
\mathbf{M}\end{array}$ & $\begin{array}{r}\text { Aggradation } \\
\left(\boldsymbol{A}_{v f}\right)\end{array}$ & $\begin{array}{r}\text { Erosion } \\
\left(\boldsymbol{E}_{v f f}\right)\end{array}$ & $\begin{array}{r}\text { Bank erosion } \\
\left(E_{b}\right)\end{array}$ & $\begin{array}{r}\text { Bank deposits } \\
\left(A_{b}\right)\end{array}$ & $\begin{array}{r}\text { Bank contribution } \\
\left(V_{b}\right)\end{array}$ \\
\hline 60 & 3 & 0.093 & -5.770 & -5.938 & 0.078 & 5.860 \\
\hline 90 & 4 & 0.039 & -3.997 & -1.633 & 0.003 & 1.630 \\
\hline 120 & 5 & 0.382 & -1.800 & -1.847 & 0.002 & 1.845 \\
\hline 150 & 6 & 0.684 & -1.522 & -2.689 & 0.006 & 2.682 \\
\hline 180 & 8 & 0.637 & -1.557 & -0.238 & 0.001 & 0.237 \\
\hline 210 & 9 & 0.384 & -2.268 & -1.149 & 0.041 & 1.108 \\
\hline 240 & 10 & 1.032 & -1.117 & -1.457 & 0.048 & 1.409 \\
\hline 270 & 11 & 1.007 & -2.156 & -3.332 & 0.584 & 2.749 \\
\hline 300 & 12 & 1.162 & -1.209 & -1.400 & 0.190 & 1.210 \\
\hline 330 & 13 & 0.690 & -1.655 & -0.317 & 0.006 & 0.311 \\
\hline 360 & 14 & 0.467 & -2.126 & -0.244 & 0.002 & 0.242 \\
\hline 390 & 15 & 1.069 & -1.521 & -1.018 & 0.317 & 0.701 \\
\hline 420 & 16 & 1.041 & -1.421 & -0.560 & 0.054 & 0.506 \\
\hline 450 & 17 & 0.565 & -1.373 & -1.072 & 0.025 & 1.047 \\
\hline 480 & 19 & 0.523 & -1.200 & -0.001 & 0.001 & 0.001 \\
\hline 510 & 20 & 1.084 & -1.644 & -1.654 & 0.001 & 1.653 \\
\hline 540 & 21 & 1.202 & -1.265 & -0.691 & 0.040 & 0.651 \\
\hline 570 & 22 & 0.961 & -1.241 & -0.360 & 0.007 & 0.353 \\
\hline 600 & 23 & 0.616 & -1.278 & -0.142 & 0.000 & 0.142 \\
\hline 630 & 24 & 0.730 & -0.845 & -0.188 & 0.004 & 0.184 \\
\hline 660 & 25 & 0.747 & -1.146 & -0.389 & 0.022 & 0.366 \\
\hline 690 & 26 & 0.547 & -1.083 & -0.553 & 0.026 & 0.527 \\
\hline
\end{tabular}

\section{Whole system}

\begin{tabular}{|c|c|c|c|c|c|c|c|}
\hline \multirow{2}{*}{ Run time } & \multirow{2}{*}{ DEM } & \multirow{2}{*}{ Aggradation $\left(A_{v f}\right)$} & \multirow{2}{*}{ Erosion $\left(E_{v f}\right)$} & \multirow{2}{*}{ Bank erosion $\left(E_{b}\right)$} & \multirow{2}{*}{ Bank deposits $\left(A_{b}\right)$} & \multicolumn{2}{|c|}{ Bank contribution } \\
\hline & & & & & & $\left(V_{b}\right)$ & $(\%)$ \\
\hline 60 & 3 & 1.696 & -10.882 & -9.342 & 0.078 & 9.263 & 50.2 \\
\hline 90 & 4 & 0.542 & -8.893 & -3.871 & 0.007 & 3.864 & 31.6 \\
\hline 120 & 5 & 0.860 & -4.679 & -4.821 & 0.013 & 4.808 & 55.7 \\
\hline 150 & 6 & 1.092 & -5.579 & -4.113 & 0.007 & 4.105 & 47.8 \\
\hline 180 & 8 & 1.057 & -4.601 & -0.902 & 0.003 & 0.899 & 20.2 \\
\hline 210 & 9 & 0.665 & -6.133 & -1.414 & 0.049 & 1.365 & 20.0 \\
\hline 240 & 10 & 2.136 & -3.888 & -3.288 & 0.059 & 3.228 & 64.8 \\
\hline 270 & 11 & 2.308 & -4.242 & -4.697 & 0.590 & 4.107 & 68.0 \\
\hline 300 & 12 & 1.815 & -3.859 & -3.269 & 0.299 & 2.971 & 59.2 \\
\hline 330 & 13 & 1.506 & -4.774 & -1.270 & 0.014 & 1.256 & 27.8 \\
\hline 360 & 14 & 0.763 & -5.297 & -0.663 & 0.097 & 0.566 & 11.1 \\
\hline 390 & 15 & 1.946 & -3.793 & -1.900 & 0.349 & 1.550 & 45.6 \\
\hline 420 & 16 & 1.816 & -3.508 & -1.012 & 0.056 & 0.956 & 36.1 \\
\hline 450 & 17 & 1.350 & -3.243 & -1.607 & 0.128 & 1.479 & 43.9 \\
\hline 480 & 19 & 1.501 & -2.403 & -0.213 & 0.031 & 0.182 & 16.8 \\
\hline 510 & 20 & 1.814 & -2.819 & -1.814 & 0.011 & 1.804 & 64.2 \\
\hline 540 & 21 & 2.106 & -2.546 & -1.452 & 0.044 & 1.408 & 76.2 \\
\hline 570 & 22 & 1.540 & -3.302 & -0.953 & 0.112 & 0.841 & 32.3 \\
\hline 600 & 23 & 2.155 & -2.754 & -1.145 & 0.003 & 1.142 & 65.6 \\
\hline 630 & 24 & 1.729 & -3.008 & -1.416 & 0.437 & 0.979 & 43.4 \\
\hline 660 & 25 & 1.893 & -3.755 & -1.112 & 0.118 & 0.994 & 34.8 \\
\hline 690 & 26 & 1.556 & -2.914 & -2.055 & 0.395 & 1.660 & 55.0 \\
\hline
\end{tabular}


Table S4. T_NC1 experiment. All values are reported in $10^{-3} \mathrm{~m}^{3}$ over a period of 30 minutes (Run time is in minutes).

\begin{tabular}{|c|c|c|c|c|c|c|}
\hline \multicolumn{7}{|c|}{ Upper section } \\
\hline $\begin{array}{l}\text { Run } \\
\text { time }\end{array}$ & $\begin{array}{r}\mathbf{D E} \\
\mathbf{M} \\
\end{array}$ & $\begin{array}{r}\text { Aggradation } \\
\left(A_{v f}\right) \\
\end{array}$ & $\begin{array}{r}\text { Erosion } \\
\left(E_{v f}\right)\end{array}$ & $\begin{array}{r}\text { Bank erosion } \\
\left(E_{b}\right)\end{array}$ & $\begin{array}{r}\text { Bank deposits } \\
\left(A_{b}\right) \\
\end{array}$ & $\begin{array}{r}\text { Bank contribution } \\
\left(V_{b}\right)\end{array}$ \\
\hline 60 & 3 & 1.775 & -0.696 & -0.020 & 0.000 & 0.020 \\
\hline 90 & 4 & 1.303 & -0.715 & -0.183 & 0.000 & 0.183 \\
\hline 120 & 5 & 0.691 & -0.869 & -0.496 & 0.000 & 0.496 \\
\hline 150 & 6 & 0.386 & -1.060 & -0.706 & 0.000 & 0.706 \\
\hline 180 & 7 & 0.204 & -0.993 & -0.517 & 0.003 & 0.514 \\
\hline 210 & 8 & 0.162 & -2.010 & -0.696 & 0.000 & 0.695 \\
\hline 240 & 9 & 0.282 & -1.653 & -0.400 & 0.000 & 0.400 \\
\hline 270 & 10 & 0.247 & -1.301 & -0.436 & 0.000 & 0.436 \\
\hline 300 & 11 & 0.175 & -0.521 & -1.426 & 0.000 & 1.426 \\
\hline 330 & 12 & 0.221 & -1.362 & -1.407 & 0.001 & 1.407 \\
\hline 360 & 14 & 0.424 & -1.809 & -2.210 & 0.002 & 2.208 \\
\hline 390 & 15 & 0.333 & -2.045 & -0.283 & 0.002 & 0.281 \\
\hline 420 & 16 & 0.240 & -2.095 & -0.137 & 0.004 & 0.134 \\
\hline 450 & 17 & 0.037 & -2.155 & -0.013 & 0.019 & -0.005 \\
\hline 480 & 18 & 0.176 & -2.328 & -0.853 & 0.123 & 0.729 \\
\hline 510 & 19 & 0.293 & -2.059 & -0.303 & 0.005 & 0.299 \\
\hline 540 & 20 & 0.106 & -2.333 & -0.003 & 0.000 & 0.003 \\
\hline 570 & 21 & 0.226 & -1.495 & -0.002 & 0.001 & 0.001 \\
\hline 600 & 22 & 0.122 & -1.399 & -0.080 & 0.002 & 0.078 \\
\hline \multicolumn{7}{|c|}{ Middle section } \\
\hline $\begin{array}{l}\text { Run } \\
\text { time }\end{array}$ & $\begin{array}{r}\mathbf{D E} \\
\mathbf{M}\end{array}$ & $\begin{array}{r}\text { Aggradation } \\
\left(A_{v f}\right)\end{array}$ & $\begin{array}{r}\text { Erosion } \\
\left(\boldsymbol{E}_{v f}\right) \\
\end{array}$ & $\begin{array}{r}\text { Bank erosion } \\
\left(E_{b}\right)\end{array}$ & $\begin{array}{r}\text { Bank deposits } \\
\left(A_{b}\right) \\
\end{array}$ & $\begin{array}{r}\text { Bank contribution } \\
\left(V_{b}\right)\end{array}$ \\
\hline 60 & 3 & 0.210 & -3.311 & -12.683 & 0.000 & 12.683 \\
\hline 90 & 4 & 0.208 & -3.819 & -3.665 & 0.000 & 3.665 \\
\hline 120 & 5 & 0.236 & -3.086 & -2.456 & 0.002 & 2.454 \\
\hline 150 & 6 & 0.360 & -2.600 & -1.102 & 0.009 & 1.093 \\
\hline 180 & 7 & 0.431 & -2.372 & -1.178 & 0.269 & 0.909 \\
\hline 210 & 8 & 0.288 & -5.014 & -0.860 & 0.065 & 0.795 \\
\hline 240 & 9 & 0.242 & -2.959 & -1.455 & 0.005 & 1.451 \\
\hline 270 & 10 & 0.470 & -4.149 & -1.629 & 0.002 & 1.626 \\
\hline 300 & 11 & 0.406 & -2.279 & -0.372 & 0.004 & 0.367 \\
\hline 330 & 12 & 0.166 & -3.429 & -2.012 & 0.002 & 2.010 \\
\hline 360 & 14 & 1.205 & -2.012 & -1.526 & 0.004 & 1.522 \\
\hline 390 & 15 & 0.229 & -4.455 & -1.599 & 0.003 & 1.596 \\
\hline 420 & 16 & 0.158 & -3.501 & -0.350 & 0.002 & 0.348 \\
\hline 450 & 17 & 0.977 & -2.187 & -0.579 & 0.005 & 0.573 \\
\hline 480 & 18 & 0.490 & -3.108 & -0.573 & 0.003 & 0.570 \\
\hline 510 & 19 & 0.809 & -1.400 & -0.270 & 0.003 & 0.267 \\
\hline 540 & 20 & 1.487 & -1.411 & -0.672 & 0.050 & 0.623 \\
\hline 570 & 21 & 0.481 & -2.106 & -0.372 & 0.003 & 0.369 \\
\hline 600 & 22 & 0.979 & -1.921 & -0.910 & 0.008 & 0.902 \\
\hline \multicolumn{7}{|c|}{ Lower section } \\
\hline $\begin{array}{l}\text { Run } \\
\text { time }\end{array}$ & $\begin{array}{r}\mathbf{D E} \\
\mathbf{M}\end{array}$ & $\begin{array}{r}\text { Aggradation } \\
\left(A_{v f}\right)\end{array}$ & $\begin{array}{r}\text { Erosion } \\
\left(\boldsymbol{E}_{v f}\right)\end{array}$ & $\begin{array}{r}\text { Bank erosion } \\
\left(E_{b}\right)\end{array}$ & $\begin{array}{r}\text { Bank deposits } \\
\left(A_{b}\right)\end{array}$ & $\begin{array}{r}\text { Bank contribution } \\
\left(V_{b}\right)\end{array}$ \\
\hline 60 & 3 & 0.016 & -5.110 & -12.381 & 0.016 & 12.364 \\
\hline 90 & 4 & 0.990 & -1.676 & -15.225 & 0.012 & 15.213 \\
\hline 120 & 5 & 0.729 & -1.493 & -16.743 & 0.122 & 16.621 \\
\hline 150 & 6 & 0.252 & -2.875 & -11.660 & 0.238 & 11.422 \\
\hline 180 & 7 & 0.640 & -2.737 & -9.402 & 0.038 & 9.364 \\
\hline
\end{tabular}




\begin{tabular}{|c|c|c|c|c|c|c|}
\hline 210 & 8 & 0.231 & -5.420 & -5.717 & 0.041 & 5.676 \\
\hline 240 & 9 & 1.036 & -2.232 & -10.947 & 0.013 & 10.935 \\
\hline 270 & 10 & 1.030 & -3.369 & -4.215 & 0.118 & 4.097 \\
\hline 300 & 11 & 1.563 & -2.496 & -9.180 & 1.431 & 7.749 \\
\hline 330 & 12 & 0.479 & -3.487 & -4.139 & 0.183 & 3.956 \\
\hline 360 & 14 & 1.241 & -1.945 & -4.929 & 3.103 & 1.826 \\
\hline 390 & 15 & 1.139 & -3.009 & -4.460 & 0.048 & 4.413 \\
\hline 420 & 16 & 0.999 & -3.683 & -0.612 & 0.016 & 0.596 \\
\hline 450 & 17 & 1.268 & -2.914 & -0.472 & 0.032 & 0.439 \\
\hline 480 & 18 & 0.929 & -1.683 & -0.016 & 0.026 & -0.010 \\
\hline 510 & 19 & 1.238 & -1.378 & -0.013 & 0.028 & -0.016 \\
\hline 540 & 20 & 1.687 & -1.924 & -0.089 & 0.021 & 0.068 \\
\hline 570 & 21 & 1.092 & -1.472 & -0.014 & 0.026 & -0.013 \\
\hline 600 & 22 & 0.558 & -2.424 & -0.037 & 0.020 & 0.018 \\
\hline
\end{tabular}

\section{Whole system}

\begin{tabular}{|r|r|r|r|r|r|r|r|}
\hline \multirow{2}{*}{ Run time } & \multirow{2}{*}{ DEM } & \multirow{2}{*}{ Aggradation $\left(\boldsymbol{A}_{\boldsymbol{v} f}\right)$} & \multirow{2}{*}{ Erosion $\left(\boldsymbol{E}_{\boldsymbol{v f}}\right)$} & \multirow{2}{*}{ Bank erosion $\left(\boldsymbol{E}_{\boldsymbol{b}}\right)$} & \multirow{2}{*}{ Bank deposits $\left(\boldsymbol{A}_{\boldsymbol{b}}\right)$} & \multicolumn{2}{|c|}{ Bank contribution } \\
\cline { 5 - 8 } & & 2.116 & -9.359 & -26.362 & 0.016 & 26.346 & 78.4 \\
\hline 60 & 3 & 2.650 & -6.327 & -20.389 & 0.012 & 20.376 & 84.7 \\
\hline 90 & 4 & 1.846 & -5.586 & -19.792 & 0.126 & 19.665 & 84.0 \\
\hline 120 & 5 & 1.132 & -6.659 & -14.314 & 0.515 & 13.799 & 71.4 \\
\hline 150 & 6 & 1.414 & -6.224 & -11.688 & 0.311 & 11.377 & 70.3 \\
\hline 180 & 7 & 0.719 & -12.803 & -7.295 & 0.108 & 7.187 & 37.3 \\
\hline 210 & 8 & 1.648 & -7.054 & -12.811 & 0.030 & 12.781 & 70.3 \\
\hline 240 & 9 & 1.905 & -9.027 & -6.684 & 0.135 & 6.548 & 47.9 \\
\hline 270 & 10 & 2.179 & -5.586 & -11.238 & 1.437 & 9.801 & 74.2 \\
\hline 300 & 11 & 0.990 & -8.393 & -8.373 & 0.246 & 8.127 & 52.3 \\
\hline 330 & 12 & 2.910 & -6.167 & -10.165 & 3.545 & 6.620 & 67.0 \\
\hline 360 & 14 & 2.134 & -9.640 & -7.248 & 0.054 & 7.194 & 48.9 \\
\hline 390 & 15 & 1.441 & -9.745 & -1.523 & 0.023 & 1.500 & 15.3 \\
\hline 420 & 16 & 2.430 & -7.576 & -1.154 & 0.064 & 1.089 & 17.5 \\
\hline 450 & 17 & 1.713 & -7.309 & -1.884 & 0.157 & 1.727 & 23.6 \\
\hline 480 & 18 & 2.420 & -5.252 & -0.711 & 0.040 & 0.672 & 19.2 \\
\hline 510 & 19 & 3.397 & -5.965 & -0.766 & 0.073 & 0.694 & 21.3 \\
\hline 540 & 20 & 1.971 & -5.141 & -0.389 & 0.032 & 0.357 & 10.1 \\
\hline 570 & 21 & 1.721 & -5.879 & -1.030 & 0.031 & 0.999 & 19.4 \\
\hline 600 & 22 & & & & &
\end{tabular}

Table S5. T_ISDS experiment. All values are reported in $10^{-3} \mathrm{~m}^{3}$ over a period of 30 minutes (Run time is in minutes).

\begin{tabular}{|c|c|c|c|c|c|c|}
\hline \multicolumn{7}{|c|}{ Upper section } \\
\hline $\begin{array}{l}\text { Run } \\
\text { time }\end{array}$ & $\begin{array}{r}\mathbf{D E} \\
\mathbf{M} \\
\end{array}$ & $\begin{array}{r}\text { Aggradation } \\
\left(A_{v f}\right) \\
\end{array}$ & $\begin{array}{r}\text { Erosion } \\
\left(E_{v f}\right) \\
\end{array}$ & $\begin{array}{r}\text { Bank erosion } \\
\left(E_{b}\right) \\
\end{array}$ & $\begin{array}{r}\text { Bank deposits } \\
\left(A_{b}\right) \\
\end{array}$ & $\begin{array}{r}\text { Bank contribution } \\
\left(V_{b}\right)\end{array}$ \\
\hline 60 & 3 & 1.733 & -0.651 & -0.024 & 0.000 & 0.024 \\
\hline 90 & 4 & 1.656 & -0.833 & -0.085 & 0.000 & 0.085 \\
\hline 120 & 5 & 1.032 & -0.871 & -0.420 & 0.000 & 0.420 \\
\hline 150 & 6 & 0.779 & -1.094 & -1.308 & 0.000 & 1.308 \\
\hline 180 & 7 & 0.817 & -0.813 & -1.170 & 0.000 & 1.170 \\
\hline 210 & 9 & 0.272 & -2.324 & -0.935 & 0.001 & 0.933 \\
\hline 240 & 11 & 0.522 & -1.055 & -0.878 & 0.006 & 0.872 \\
\hline 270 & 12 & 0.495 & -0.692 & -0.521 & 0.001 & 0.521 \\
\hline 300 & 13 & 0.337 & -1.154 & -0.818 & 0.003 & 0.815 \\
\hline 330 & 14 & 0.125 & -1.516 & -2.384 & 0.008 & 2.376 \\
\hline 360 & 15 & 0.022 & -1.806 & -0.187 & 0.001 & 0.186 \\
\hline
\end{tabular}




\begin{tabular}{|c|c|c|c|c|c|c|}
\hline 390 & 16 & 0.097 & -1.319 & -0.052 & 0.035 & 0.017 \\
\hline 420 & 17 & 0.186 & -0.552 & -0.562 & 0.001 & 0.561 \\
\hline 450 & 19 & 0.458 & -0.814 & -0.583 & 0.006 & 0.577 \\
\hline 480 & 20 & 0.572 & -1.226 & -0.523 & 0.030 & 0.493 \\
\hline 510 & 21 & 0.246 & -2.032 & -1.472 & 0.271 & 1.201 \\
\hline 540 & 22 & 0.745 & -1.731 & -1.299 & 0.055 & 1.244 \\
\hline 570 & 23 & 0.471 & -0.977 & -1.172 & 0.001 & 1.171 \\
\hline 600 & 24 & 0.836 & -1.499 & -0.311 & 0.078 & 0.232 \\
\hline 630 & 25 & 0.304 & -0.665 & -0.362 & 0.018 & 0.345 \\
\hline 660 & 26 & 0.408 & -1.371 & -0.256 & 0.001 & 0.255 \\
\hline 690 & 27 & 0.734 & -1.060 & -0.027 & 0.005 & 0.022 \\
\hline 720 & 28 & 0.316 & -0.659 & -0.883 & 0.301 & 0.582 \\
\hline \multicolumn{7}{|c|}{ Middle section } \\
\hline $\begin{array}{l}\text { Run } \\
\text { time }\end{array}$ & $\begin{array}{r}\mathbf{D E} \\
\mathbf{M} \\
\end{array}$ & $\begin{array}{r}\text { Aggradation } \\
\left(A_{v f}\right) \\
\end{array}$ & $\begin{array}{r}\text { Erosion } \\
\left(E_{v f}\right) \\
\end{array}$ & $\begin{array}{r}\text { Bank erosion } \\
\left(E_{b}\right) \\
\end{array}$ & $\begin{array}{r}\text { Bank deposits } \\
\left(A_{b}\right) \\
\end{array}$ & $\begin{array}{r}\text { Bank contribution } \\
\left(V_{b}\right)\end{array}$ \\
\hline 60 & 3 & 1.558 & -1.489 & -13.991 & 0.000 & 13.991 \\
\hline 90 & 4 & 0.168 & -5.316 & -7.828 & 0.027 & 7.802 \\
\hline 120 & 5 & 0.239 & -2.597 & -4.204 & 0.001 & 4.203 \\
\hline 150 & 6 & 0.961 & -1.566 & -4.134 & 0.011 & 4.123 \\
\hline 180 & 7 & 0.561 & -4.485 & -1.829 & 0.278 & 1.551 \\
\hline 210 & 9 & 1.462 & -3.040 & -2.131 & 0.018 & 2.113 \\
\hline 240 & 11 & 1.708 & -2.441 & -0.496 & 0.278 & 0.218 \\
\hline 270 & 12 & 0.583 & -3.631 & -0.980 & 0.504 & 0.476 \\
\hline 300 & 13 & 0.545 & -3.996 & -1.543 & 0.019 & 1.524 \\
\hline 330 & 14 & 1.169 & -2.262 & -0.717 & 0.048 & 0.669 \\
\hline 360 & 15 & 1.696 & -3.458 & -0.038 & 0.003 & 0.035 \\
\hline 390 & 16 & 3.359 & -1.155 & -0.674 & 0.002 & 0.672 \\
\hline 420 & 17 & 3.106 & -1.109 & -0.707 & 0.017 & 0.689 \\
\hline 450 & 19 & 3.016 & -1.115 & -1.412 & 0.747 & 0.665 \\
\hline 480 & 20 & 2.730 & -2.975 & -2.446 & 0.239 & 2.208 \\
\hline 510 & 21 & 0.907 & -2.679 & -1.275 & 0.054 & 1.221 \\
\hline 540 & 22 & 1.457 & -2.017 & -0.354 & 0.003 & 0.351 \\
\hline 570 & 23 & 1.847 & -2.646 & -3.076 & 0.744 & 2.332 \\
\hline 600 & 24 & 1.322 & -3.256 & -2.434 & 0.002 & 2.432 \\
\hline 630 & 25 & 0.665 & -2.032 & -2.671 & 0.032 & 2.640 \\
\hline 660 & 26 & 1.383 & -2.565 & -0.560 & 0.017 & 0.544 \\
\hline 690 & 27 & 1.695 & -2.410 & -2.386 & 0.002 & 2.384 \\
\hline 720 & 28 & 1.505 & -4.083 & -0.406 & 0.125 & 0.281 \\
\hline \multicolumn{7}{|c|}{ Lower section } \\
\hline $\begin{array}{l}\text { Run } \\
\text { time }\end{array}$ & $\begin{array}{r}\mathbf{D E} \\
\mathbf{M} \\
\end{array}$ & $\begin{array}{r}\text { Aggradation } \\
\left(A_{v f}\right) \\
\end{array}$ & $\begin{array}{r}\text { Erosion } \\
\left(E_{v f}\right) \\
\end{array}$ & $\begin{array}{r}\text { Bank erosion } \\
\left(E_{b}\right) \\
\end{array}$ & $\begin{array}{r}\text { Bank deposits } \\
\left(A_{b}\right) \\
\end{array}$ & $\begin{array}{r}\text { Bank contribution } \\
\left(V_{b}\right)\end{array}$ \\
\hline 60 & 3 & 1.450 & -1.853 & -16.482 & 0.000 & 16.482 \\
\hline 90 & 4 & 0.516 & -4.613 & -11.784 & 1.038 & 10.746 \\
\hline 120 & 5 & 0.583 & -3.192 & -9.337 & 0.398 & 8.938 \\
\hline 150 & 6 & 1.347 & -2.221 & -8.146 & 0.173 & 7.973 \\
\hline 180 & 7 & 0.657 & -4.074 & -8.794 & 3.060 & 5.734 \\
\hline 210 & 9 & 1.448 & -1.525 & -9.024 & 2.001 & 7.023 \\
\hline 240 & 11 & 2.665 & -1.447 & -12.243 & 0.555 & 11.688 \\
\hline 270 & 12 & 0.532 & -4.877 & -5.675 & 0.007 & 5.668 \\
\hline 300 & 13 & 0.840 & -2.245 & -4.870 & 2.504 & 2.366 \\
\hline 330 & 14 & 1.721 & -1.620 & -6.417 & 0.068 & 6.349 \\
\hline 360 & 15 & 0.659 & -4.546 & -0.907 & 0.012 & 0.894 \\
\hline 390 & 16 & 1.238 & -2.528 & -5.398 & 2.270 & 3.128 \\
\hline 420 & 17 & 1.287 & -2.107 & -2.920 & 0.010 & 2.910 \\
\hline 450 & 19 & 3.109 & -0.640 & -7.602 & 2.356 & 5.247 \\
\hline 480 & 20 & 0.228 & -4.964 & -1.137 & 0.007 & 1.130 \\
\hline 510 & 21 & 1.449 & -2.011 & -2.039 & 0.007 & 2.031 \\
\hline 540 & 22 & 1.778 & -2.432 & -1.376 & 0.014 & 1.363 \\
\hline 570 & 23 & 2.092 & -1.620 & -9.518 & 4.648 & 4.870 \\
\hline
\end{tabular}




\begin{tabular}{|l|l|l|l|l|l|r|}
\hline 600 & 24 & 1.876 & -2.551 & -6.514 & 2.266 & 4.248 \\
\hline 630 & 25 & 0.838 & -3.997 & -3.124 & 0.009 & 3.115 \\
\hline 660 & 26 & 1.224 & -2.177 & -2.396 & 0.007 & 2.389 \\
\hline 690 & 27 & 1.993 & -2.429 & -1.264 & 0.010 & 1.254 \\
\hline 720 & 28 & 1.068 & -2.940 & -4.726 & 1.884 & 2.841 \\
\hline
\end{tabular}

\section{Whole system}

\begin{tabular}{|c|c|c|c|c|c|c|c|}
\hline \multirow{2}{*}{ Run time } & \multirow{2}{*}{ DEM } & \multirow{2}{*}{ Aggradation $\left(A_{v f}\right)$} & \multirow{2}{*}{ Erosion $\left(E_{v f}\right)$} & \multirow{2}{*}{ Bank erosion $\left(E_{b}\right)$} & \multirow{2}{*}{ Bank deposits $\left(A_{b}\right)$} & \multicolumn{2}{|c|}{ Bank contribution } \\
\hline & & & & & & $\left(V_{b}\right)$ & $(\%)$ \\
\hline 60 & 3 & 4.917 & -4.199 & -31.161 & 0.000 & 31.161 & 102.4 \\
\hline 90 & 4 & 2.486 & -11.114 & -20.217 & 1.065 & 19.153 & 68.9 \\
\hline 120 & 5 & 1.989 & -7.102 & -14.506 & 0.400 & 14.107 & 73.4 \\
\hline 150 & 6 & 3.340 & -4.981 & -14.580 & 0.184 & 14.395 & 89.8 \\
\hline 180 & 7 & 2.110 & -9.482 & -11.797 & 3.342 & 8.455 & 53.4 \\
\hline 210 & 9 & 3.270 & -7.124 & -12.104 & 2.024 & 10.080 & 72.3 \\
\hline 240 & 11 & 5.153 & -5.084 & -14.680 & 1.154 & 13.526 & 100.5 \\
\hline 270 & 12 & 1.856 & -9.462 & -7.576 & 0.514 & 7.062 & 48.1 \\
\hline 300 & 13 & 1.776 & -7.654 & -7.233 & 2.526 & 4.707 & 44.5 \\
\hline 330 & 14 & 3.137 & -5.561 & -9.672 & 0.125 & 9.547 & 79.7 \\
\hline 360 & 15 & 2.393 & -10.168 & -1.154 & 0.017 & 1.137 & 12.8 \\
\hline 390 & 16 & 4.872 & -5.081 & -6.139 & 2.308 & 3.831 & 94.8 \\
\hline 420 & 17 & 4.610 & -4.007 & -4.189 & 0.030 & 4.160 & 117.0 \\
\hline 450 & 19 & 6.939 & -2.698 & -9.695 & 3.109 & 6.586 & 280.9 \\
\hline 480 & 20 & 3.587 & -9.543 & -4.111 & 0.277 & 3.834 & 39.2 \\
\hline 510 & 21 & 2.749 & -6.897 & -4.794 & 0.337 & 4.458 & 51.8 \\
\hline 540 & 22 & 4.023 & -6.526 & -3.310 & 0.073 & 3.237 & 56.4 \\
\hline 570 & 23 & 4.593 & -5.411 & -13.926 & 5.394 & 8.531 & 91.3 \\
\hline 600 & 24 & 4.237 & -7.462 & -9.279 & 2.349 & 6.930 & 68.2 \\
\hline 630 & 25 & 1.889 & -6.840 & -6.168 & 0.059 & 6.108 & 55.2 \\
\hline 660 & 26 & 3.170 & -6.284 & -3.221 & 0.026 & 3.195 & 50.6 \\
\hline 690 & 27 & 4.533 & -6.040 & -4.432 & 0.019 & 4.414 & 74.6 \\
\hline 720 & 28 & 2.975 & -7.930 & -7.218 & 2.530 & 4.688 & 48.6 \\
\hline
\end{tabular}

Table S6. T_DWIW experiment. All values are reported in $10^{-3} \mathrm{~m}^{3}$ over a period of 30 minutes (Run time is in minutes).

\begin{tabular}{|c|c|c|c|c|c|c|}
\hline \multicolumn{7}{|c|}{ Upper section } \\
\hline $\begin{array}{l}\text { Run } \\
\text { time }\end{array}$ & $\begin{array}{r}\mathbf{D E} \\
\mathbf{M}\end{array}$ & $\begin{array}{r}\text { Aggradation } \\
\left(A_{v f}\right)\end{array}$ & $\begin{array}{r}\text { Erosion } \\
\left(E_{v f}\right)\end{array}$ & $\begin{array}{r}\text { Bank erosion } \\
\left(E_{b}\right)\end{array}$ & $\begin{array}{r}\text { Bank deposits } \\
\left(A_{b}\right)\end{array}$ & $\begin{array}{r}\text { Bank contribution } \\
\left(V_{b}\right)\end{array}$ \\
\hline 60 & 3 & 1.048 & -1.367 & -0.012 & 0.000 & 0.012 \\
\hline 90 & 4 & 0.767 & -0.970 & -0.248 & 0.000 & 0.248 \\
\hline 120 & 5 & 0.482 & -0.852 & -0.531 & 0.000 & 0.531 \\
\hline 150 & 6 & 0.195 & -1.327 & -0.369 & 0.000 & 0.369 \\
\hline 180 & 7 & 0.170 & -1.581 & -0.457 & 0.000 & 0.456 \\
\hline 210 & 8 & 0.160 & -1.071 & -0.410 & 0.001 & 0.409 \\
\hline 240 & 9 & 0.541 & -1.699 & -0.242 & 0.000 & 0.242 \\
\hline 270 & 10 & 0.035 & -2.822 & -0.724 & 0.000 & 0.724 \\
\hline 300 & 11 & 0.090 & -1.624 & -0.282 & 0.000 & 0.282 \\
\hline 330 & 13 & 0.036 & -1.879 & -0.102 & 0.001 & 0.102 \\
\hline 360 & 14 & 0.133 & -1.412 & -0.001 & 0.000 & 0.001 \\
\hline 390 & 15 & 0.217 & -0.769 & -0.009 & 0.001 & 0.008 \\
\hline 420 & 16 & 0.600 & -0.550 & -0.369 & 0.003 & 0.366 \\
\hline 450 & 17 & 0.552 & -0.874 & -1.134 & 0.009 & 1.125 \\
\hline 480 & 18 & 0.620 & -1.779 & -0.089 & 0.000 & 0.089 \\
\hline 510 & 19 & 0.077 & -1.221 & -0.083 & 0.000 & 0.083 \\
\hline
\end{tabular}




\begin{tabular}{|c|c|c|c|c|c|c|}
\hline 540 & 20 & 0.563 & -1.709 & -0.062 & 0.000 & 0.062 \\
\hline 570 & 21 & 0.590 & -1.606 & -0.139 & 0.000 & 0.139 \\
\hline 600 & 22 & 0.732 & -0.602 & -0.446 & 0.000 & 0.446 \\
\hline 630 & 23 & 0.157 & -0.717 & -0.051 & 0.037 & 0.014 \\
\hline 660 & 24 & 0.370 & -0.804 & -0.109 & 0.002 & 0.107 \\
\hline 690 & 25 & 0.052 & -0.675 & -0.071 & 0.000 & 0.071 \\
\hline \multicolumn{7}{|c|}{ Middle section } \\
\hline $\begin{array}{l}\text { Run } \\
\text { time }\end{array}$ & $\begin{array}{r}\mathbf{D E} \\
\mathbf{M}\end{array}$ & $\begin{array}{r}\text { Aggradation } \\
\left(A_{v f}\right)\end{array}$ & $\begin{array}{r}\text { Erosion } \\
\left(\boldsymbol{E}_{v f}\right) \\
\end{array}$ & $\begin{array}{r}\text { Bank erosion } \\
\left(E_{b}\right) \\
\end{array}$ & $\begin{array}{r}\text { Bank deposits } \\
\left(A_{b}\right) \\
\end{array}$ & $\begin{array}{r}\text { Bank contribution } \\
\left(V_{b}\right)\end{array}$ \\
\hline 60 & 3 & 0.635 & -3.560 & -5.567 & 0.000 & 5.567 \\
\hline 90 & 4 & 0.689 & -2.503 & -4.365 & 0.000 & 4.365 \\
\hline 120 & 5 & 0.670 & -1.847 & -6.316 & 0.000 & 6.316 \\
\hline 150 & 6 & 0.467 & -2.802 & -1.306 & 0.161 & 1.144 \\
\hline 180 & 7 & 0.395 & -2.856 & -1.483 & 0.036 & 1.447 \\
\hline 210 & 8 & 0.256 & -3.247 & -0.085 & 0.020 & 0.065 \\
\hline 240 & 9 & 0.721 & -2.469 & -0.665 & 0.018 & 0.647 \\
\hline 270 & 10 & 0.152 & -4.597 & -0.144 & 0.003 & 0.141 \\
\hline 300 & 11 & 0.590 & -4.727 & -0.323 & 0.009 & 0.314 \\
\hline 330 & 13 & 1.280 & -1.845 & -1.464 & 0.125 & 1.339 \\
\hline 360 & 14 & 1.750 & -1.802 & -0.316 & 0.004 & 0.312 \\
\hline 390 & 15 & 0.959 & -0.861 & -0.010 & 0.012 & -0.002 \\
\hline 420 & 16 & 2.326 & -1.442 & -3.676 & 0.141 & 3.535 \\
\hline 450 & 17 & 2.715 & -2.749 & -6.680 & 0.014 & 6.666 \\
\hline 480 & 18 & 0.557 & -3.791 & -1.448 & 0.003 & 1.446 \\
\hline 510 & 19 & 1.019 & -3.313 & -4.474 & 0.022 & 4.451 \\
\hline 540 & 20 & 0.853 & -4.344 & -2.725 & 0.161 & 2.564 \\
\hline 570 & 21 & 1.813 & -3.366 & -5.011 & 1.800 & 3.210 \\
\hline 600 & 22 & 1.095 & -3.360 & -3.059 & 0.003 & 3.056 \\
\hline 630 & 23 & 1.142 & -2.503 & -0.320 & 1.452 & -1.132 \\
\hline 660 & 24 & 2.158 & -2.246 & -7.080 & 1.934 & 5.146 \\
\hline 690 & 25 & 1.103 & -3.125 & -0.284 & 0.016 & 0.268 \\
\hline \multicolumn{7}{|c|}{ Lower section } \\
\hline $\begin{array}{l}\text { Run } \\
\text { time }\end{array}$ & $\begin{array}{r}\mathbf{D E} \\
\mathbf{M}\end{array}$ & $\begin{array}{r}\text { Aggradation } \\
\left(A_{v f}\right)\end{array}$ & $\begin{array}{r}\text { Erosion } \\
\left(\boldsymbol{E}_{v f}\right)\end{array}$ & $\begin{array}{r}\text { Bank erosion } \\
\left(E_{b}\right)\end{array}$ & $\begin{array}{r}\text { Bank deposits } \\
\left(A_{b}\right)\end{array}$ & $\begin{array}{r}\text { Bank contribution } \\
\left(V_{b}\right)\end{array}$ \\
\hline 60 & 3 & 0.129 & -3.326 & -11.569 & 0.020 & 11.549 \\
\hline 90 & 4 & 0.733 & -2.896 & -17.922 & 0.006 & 17.916 \\
\hline 120 & 5 & 0.361 & -4.085 & -4.880 & 0.179 & 4.701 \\
\hline 150 & 6 & 0.300 & -5.205 & -8.545 & 1.094 & 7.451 \\
\hline 180 & 7 & 2.931 & -1.254 & -11.580 & 2.407 & 9.173 \\
\hline 210 & 8 & 0.555 & -2.438 & -9.934 & 0.232 & 9.701 \\
\hline 240 & 9 & 1.106 & -3.421 & -10.849 & 0.187 & 10.662 \\
\hline 270 & 10 & 0.321 & -4.213 & -7.876 & 0.006 & 7.871 \\
\hline 300 & 11 & 0.786 & -3.940 & $\begin{array}{l}-10.673 \\
\end{array}$ & 4.263 & 6.410 \\
\hline 330 & 13 & 1.119 & -2.037 & -4.303 & 0.037 & 4.266 \\
\hline 360 & 14 & 0.219 & -2.342 & -2.478 & 0.009 & 2.469 \\
\hline 390 & 15 & 0.995 & -1.824 & -3.870 & 0.675 & 3.195 \\
\hline 420 & 16 & 1.087 & -2.459 & -2.617 & 0.002 & 2.614 \\
\hline 450 & 17 & 1.577 & -1.377 & -0.399 & 0.024 & 0.376 \\
\hline 480 & 18 & 0.918 & -2.979 & -1.209 & 0.692 & 0.517 \\
\hline 510 & 19 & 1.411 & -1.463 & -9.344 & 5.989 & 3.355 \\
\hline 540 & 20 & 1.112 & -2.042 & -3.908 & 0.064 & 3.843 \\
\hline 570 & 21 & 1.388 & -2.530 & -2.060 & 0.009 & 2.051 \\
\hline 600 & 22 & 0.927 & -2.390 & -1.937 & 0.009 & 1.927 \\
\hline 630 & 23 & 1.145 & -1.537 & -0.167 & 0.017 & 0.150 \\
\hline 660 & 24 & 0.710 & -2.831 & -0.434 & 0.022 & 0.412 \\
\hline 690 & 25 & 2.649 & -0.838 & -3.556 & 0.109 & 3.447 \\
\hline
\end{tabular}

\section{Whole system}




\begin{tabular}{|r|r|r|r|r|r|r|r|}
\hline \multirow{2}{*}{ Run time } & DEM & Aggradation $\left(\boldsymbol{A}_{\boldsymbol{v} f}\right)$ & Erosion $\left(\boldsymbol{E}_{\boldsymbol{v} f}\right)$ & Bank erosion $\left(\boldsymbol{E}_{\boldsymbol{b}}\right)$ & Bank deposits $\left(\boldsymbol{A}_{\boldsymbol{b}}\right)$ & \multicolumn{2}{|c|}{ Bank contribution } \\
\hline & & 1.990 & -8.639 & -18.494 & 0.021 & 18.474 & 73.5 \\
\hline 60 & 3 & 2.481 & -6.552 & -23.348 & 0.008 & 23.340 & 85.1 \\
\hline 90 & 4 & 1.641 & -7.143 & -12.961 & 0.333 & 12.629 & 69.7 \\
\hline 120 & 5 & 1.007 & -9.587 & -10.979 & 1.260 & 9.719 & 53.1 \\
\hline 150 & 6 & 3.919 & -5.824 & -13.791 & 2.506 & 11.285 & 85.6 \\
\hline 180 & 7 & 1.110 & -7.011 & -10.503 & 0.254 & 10.249 & 63.5 \\
\hline 210 & 8 & 2.445 & -8.028 & -11.814 & 0.207 & 11.607 & 67.5 \\
\hline 240 & 9 & 0.548 & -11.991 & -8.753 & 0.010 & 8.743 & 43.3 \\
\hline 270 & 10 & 1.474 & -10.676 & -11.522 & 4.274 & 7.248 & 44.1 \\
\hline 300 & 11 & 2.487 & -6.004 & -5.895 & 0.164 & 5.731 & 62.0 \\
\hline 330 & 13 & 2.154 & -5.711 & -2.796 & 0.013 & 2.782 & 43.9 \\
\hline 360 & 14 & 2.306 & -3.518 & -3.890 & 0.689 & 3.201 & 72.5 \\
\hline 390 & 15 & 4.162 & -4.620 & -7.167 & 0.262 & 6.905 & 93.8 \\
\hline 420 & 16 & 4.912 & -5.253 & -8.338 & 0.047 & 8.291 & 96.1 \\
\hline 450 & 17 & 2.160 & -8.697 & -3.411 & 1.023 & 2.388 & 26.8 \\
\hline 480 & 18 & 2.658 & -6.110 & -14.341 & 6.013 & 8.328 & 70.7 \\
\hline 510 & 19 & 2.635 & -8.371 & -6.721 & 0.226 & 6.495 & 53.1 \\
\hline 540 & 20 & 3.922 & -7.791 & -7.315 & 1.810 & 5.506 & 58.7 \\
\hline 570 & 21 & 2.932 & -6.544 & -5.442 & 0.013 & 5.429 & 60.0 \\
\hline 600 & 22 & 2.515 & -4.903 & -0.538 & 1.506 & -0.968 & 68.1 \\
\hline 630 & 23 & 3.358 & -5.994 & -7.623 & 1.958 & 5.665 & 68.2 \\
\hline 660 & 24 & 3.866 & -4.765 & -3.911 & 0.125 & 3.786 & 80.8 \\
\hline 690 & 25 & & & &
\end{tabular}

Table S7. T_NC2 experiment. All values are reported in $10^{-3} \mathrm{~m}^{3}$ over a period of 30 minutes (Run time is in minutes).

\begin{tabular}{|c|c|c|c|c|c|c|}
\hline \multicolumn{7}{|c|}{ Upper section } \\
\hline $\begin{array}{l}\text { Run } \\
\text { time }\end{array}$ & $\begin{array}{r}\mathbf{D E} \\
\mathbf{M}\end{array}$ & $\begin{array}{r}\text { Aggradation } \\
\left(A_{v f}\right)\end{array}$ & $\begin{array}{r}\text { Erosion } \\
\left(\boldsymbol{E}_{v f}\right)\end{array}$ & $\begin{array}{r}\text { Bank erosion } \\
\left(E_{b}\right)\end{array}$ & $\begin{array}{r}\text { Bank deposits } \\
\left(A_{b}\right)\end{array}$ & $\begin{array}{r}\text { Bank contribution } \\
\left(V_{b}\right)\end{array}$ \\
\hline 60 & 3 & $\begin{array}{r}(A v f) \\
1.998\end{array}$ & $\begin{array}{r}(\boldsymbol{L} v f) \\
-0.480\end{array}$ & $\begin{array}{r}\left(\boldsymbol{\Sigma}_{b)}\right) \\
0.000\end{array}$ & $\begin{array}{r}(A b) \\
0.000\end{array}$ & $\begin{array}{r}(v b) \\
0.000\end{array}$ \\
\hline 90 & 5 & 2.082 & -0.435 & -0.024 & 0.000 & 0.024 \\
\hline 120 & 6 & 1.669 & -0.395 & -0.033 & 0.000 & 0.033 \\
\hline 150 & 7 & 1.324 & -0.470 & -0.006 & 0.000 & 0.006 \\
\hline 180 & 8 & 1.502 & -0.438 & -0.045 & 0.000 & 0.045 \\
\hline 210 & 9 & 1.261 & -0.337 & 0.000 & 0.000 & 0.000 \\
\hline 240 & 10 & 1.546 & -0.618 & -0.198 & 0.000 & 0.198 \\
\hline 270 & 12 & 1.311 & -0.504 & -0.066 & 0.000 & 0.066 \\
\hline 300 & 13 & 1.328 & -0.519 & -0.129 & 0.000 & 0.129 \\
\hline 330 & 14 & 1.140 & -0.452 & -0.162 & 0.000 & 0.162 \\
\hline 360 & 15 & 0.598 & -0.471 & -0.096 & 0.000 & 0.096 \\
\hline 390 & 16 & 0.944 & -0.359 & -0.013 & 0.000 & 0.013 \\
\hline 420 & 17 & 0.985 & -0.248 & 0.000 & 0.000 & 0.000 \\
\hline 450 & 18 & 0.326 & -0.494 & -0.209 & 0.000 & 0.209 \\
\hline 480 & 19 & 1.016 & -0.396 & -0.643 & 0.000 & 0.643 \\
\hline \multicolumn{7}{|c|}{ Middle section } \\
\hline $\begin{array}{l}\text { Run } \\
\text { time }\end{array}$ & $\begin{array}{r}\text { DE } \\
\text { M }\end{array}$ & $\begin{array}{r}\text { Aggradation } \\
\left(A_{v f}\right)\end{array}$ & $\begin{array}{r}\text { Erosion } \\
\left(E_{v f}\right)\end{array}$ & $\begin{array}{r}\text { Bank erosion } \\
\left(E_{b}\right)\end{array}$ & $\begin{array}{r}\text { Bank deposits } \\
\left(A_{b}\right)\end{array}$ & $\begin{array}{r}\text { Bank contribution } \\
\left(V_{b}\right)\end{array}$ \\
\hline 60 & 3 & 2.269 & -1.347 & -5.030 & 0.000 & 5.030 \\
\hline 90 & 5 & 1.734 & -1.062 & -4.686 & 0.000 & 4.686 \\
\hline 120 & 6 & 1.645 & -1.771 & -3.598 & 0.000 & 3.598 \\
\hline 150 & 7 & 2.176 & -0.926 & -2.967 & 0.488 & 2.479 \\
\hline 180 & 8 & 1.470 & -1.354 & -2.919 & 0.001 & 2.918 \\
\hline 210 & 9 & 2.533 & -0.723 & -3.288 & 0.083 & 3.205 \\
\hline 240 & 10 & 1.511 & -1.232 & -1.123 & 0.004 & 1.120 \\
\hline 270 & 12 & 2.962 & -1.525 & -1.157 & 0.059 & 1.097 \\
\hline 300 & 13 & 1.066 & -1.610 & -2.216 & 0.037 & 2.179 \\
\hline
\end{tabular}




\begin{tabular}{|c|c|c|c|c|c|c|}
\hline 330 & 14 & 1.408 & -1.019 & -1.372 & 0.053 & 1.319 \\
\hline 360 & 15 & 1.832 & -0.978 & -0.864 & 0.002 & 0.862 \\
\hline 390 & 16 & 1.567 & -1.591 & -2.137 & 0.001 & 2.136 \\
\hline 420 & 17 & 0.715 & -2.778 & -0.980 & 0.015 & 0.965 \\
\hline 450 & 18 & 1.458 & -1.811 & -1.102 & 0.010 & 1.092 \\
\hline 480 & 19 & 1.205 & -1.125 & -0.833 & 0.092 & 0.741 \\
\hline \multicolumn{7}{|c|}{ Lower section } \\
\hline $\begin{array}{l}\text { Run } \\
\text { time }\end{array}$ & $\begin{array}{r}\mathbf{D E} \\
\mathbf{M}\end{array}$ & $\begin{array}{r}\text { Aggradation } \\
\left(A_{v f}\right)\end{array}$ & $\begin{array}{r}\text { Erosion } \\
\left(E_{v f}\right) \\
\end{array}$ & $\begin{array}{r}\text { Bank erosion } \\
\left(E_{b}\right)\end{array}$ & $\begin{array}{r}\text { Bank deposits } \\
\left(A_{b}\right) \\
\end{array}$ & $\begin{array}{r}\text { Bank contribution } \\
\left(V_{b}\right)\end{array}$ \\
\hline 60 & 3 & 0.399 & -1.956 & -5.741 & 0.002 & 5.739 \\
\hline 90 & 5 & 0.330 & -2.057 & -4.739 & 0.033 & 4.706 \\
\hline 120 & 6 & 0.903 & -1.570 & -5.584 & 0.068 & 5.516 \\
\hline 150 & 7 & 0.228 & -3.140 & -2.848 & 0.018 & 2.830 \\
\hline 180 & 8 & 2.177 & -1.495 & -2.978 & 0.004 & 2.974 \\
\hline 210 & 9 & 0.958 & -2.083 & -6.084 & 0.062 & 6.022 \\
\hline 240 & 10 & 1.605 & -1.273 & -3.964 & 0.003 & 3.961 \\
\hline 270 & 12 & 2.603 & -1.233 & -3.834 & 0.158 & 3.676 \\
\hline 300 & 13 & 1.131 & -1.953 & -1.865 & 0.020 & 1.845 \\
\hline 330 & 14 & 0.858 & -2.271 & -5.217 & 0.757 & 4.460 \\
\hline 360 & 15 & 1.154 & -1.566 & -2.897 & 0.025 & 2.872 \\
\hline 390 & 16 & 1.364 & -1.261 & -5.408 & 2.125 & 3.284 \\
\hline 420 & 17 & 0.532 & -2.196 & -1.780 & 0.078 & 1.702 \\
\hline 450 & 18 & 1.515 & -1.301 & -5.371 & 0.896 & 4.475 \\
\hline 480 & 19 & 1.848 & -1.890 & -5.235 & 0.221 & 5.013 \\
\hline
\end{tabular}

\section{Whole system}

\begin{tabular}{|c|c|c|c|c|c|c|c|}
\hline \multirow{2}{*}{ Run time } & \multirow{2}{*}{ DEM } & \multirow{2}{*}{ Aggradation $\left(A_{v f}\right)$} & \multirow{2}{*}{ Erosion $\left(E_{v f}\right)$} & \multirow{2}{*}{ Bank erosion $\left(E_{b}\right)$} & \multirow{2}{*}{ Bank deposits $\left(A_{b}\right)$} & \multicolumn{2}{|c|}{ Bank contribution } \\
\hline & & & & & & $\left(V_{b}\right)$ & $(\%)$ \\
\hline 60 & 3 & 4.785 & -4.009 & -12.082 & 0.002 & 12.081 & 106.9 \\
\hline 90 & 5 & 4.303 & -3.883 & -9.753 & 0.041 & 9.713 & 104.5 \\
\hline 120 & 6 & 4.523 & -3.915 & -9.571 & 0.068 & 9.503 & 106.8 \\
\hline 150 & 7 & 3.828 & -4.807 & -6.099 & 0.507 & 5.592 & 85.1 \\
\hline 180 & 8 & 5.515 & -3.401 & -6.348 & 0.005 & 6.342 & 150.0 \\
\hline 210 & 9 & 4.853 & -3.294 & -9.418 & 0.146 & 9.273 & 120.2 \\
\hline 240 & 10 & 4.815 & -3.225 & -5.777 & 0.006 & 5.770 & 138.0 \\
\hline 270 & 12 & 7.223 & -3.300 & -5.202 & 0.218 & 4.984 & 469.9 \\
\hline 300 & 13 & 3.786 & -4.185 & -4.637 & 0.057 & 4.580 & 92.0 \\
\hline 330 & 14 & 3.710 & -4.073 & -6.769 & 0.810 & 5.958 & 94.3 \\
\hline 360 & 15 & 3.701 & -3.096 & -3.870 & 0.029 & 3.841 & 118.7 \\
\hline 390 & 16 & 3.979 & -3.298 & -7.670 & 2.187 & 5.483 & 114.2 \\
\hline 420 & 17 & 2.320 & -5.396 & -2.799 & 0.093 & 2.706 & 46.8 \\
\hline 450 & 18 & 3.390 & -3.785 & -6.686 & 0.906 & 5.780 & 93.6 \\
\hline 480 & 19 & 4.389 & -3.606 & -6.743 & 0.326 & 6.417 & 113.9 \\
\hline
\end{tabular}

Table S8. T_IWMC experiment. All values are reported in $10^{-3} \mathrm{~m}^{3}$ over a period of 30 minutes (Run time is in minutes).

\begin{tabular}{|r|r|r|r|r|r|r|}
\hline \multicolumn{2}{|l|}{ Upper section } & \multicolumn{1}{|c|}{} \\
\hline $\begin{array}{r}\text { Run } \\
\text { time }\end{array}$ & $\begin{array}{r}\text { DE } \\
\mathbf{M}\end{array}$ & $\begin{array}{r}\text { Aggradation } \\
\left(\boldsymbol{A}_{\boldsymbol{v} f}\right)\end{array}$ & $\begin{array}{r}\text { Erosion } \\
\left(\boldsymbol{E}_{\boldsymbol{v} f}\right)\end{array}$ & $\begin{array}{r}\text { Bank erosion } \\
\left(\boldsymbol{E}_{\boldsymbol{b}}\right)\end{array}$ & $\begin{array}{r}\text { Bank deposits } \\
\left(\boldsymbol{A}_{\boldsymbol{b}}\right)\end{array}$ & $\begin{array}{r}\text { Bank contribution } \\
\left(\boldsymbol{V}_{\boldsymbol{b}}\right)\end{array}$ \\
\hline 60 & 3 & 1.600 & -0.516 & 0.000 & 0.000 & 0.000 \\
\hline 90 & 4 & 1.524 & -0.553 & -0.003 & 0.000 & 0.003 \\
\hline 120 & 5 & 1.294 & -0.423 & -0.062 & 0.000 & 0.062 \\
\hline 150 & 6 & 1.320 & -0.380 & -0.001 & 0.000 & 0.001 \\
\hline 180 & 7 & 1.383 & -0.337 & -0.010 & 0.000 & 0.010 \\
\hline
\end{tabular}




\begin{tabular}{|c|c|c|c|c|c|c|}
\hline 210 & 8 & 0.495 & -2.022 & -0.499 & 0.000 & 0.499 \\
\hline 240 & 9 & 0.067 & -2.934 & -0.285 & 0.000 & 0.285 \\
\hline 270 & 11 & 0.309 & -1.496 & -0.618 & 0.000 & 0.618 \\
\hline 300 & 12 & 0.050 & -2.686 & -0.206 & 0.000 & 0.206 \\
\hline 330 & 13 & 0.243 & -1.108 & -0.097 & 0.000 & 0.097 \\
\hline 360 & 14 & 0.313 & -0.739 & -1.490 & 0.151 & 1.340 \\
\hline 390 & 15 & 0.246 & -0.838 & -2.745 & 0.003 & 2.742 \\
\hline 420 & 16 & 0.273 & -0.638 & -1.602 & 0.000 & 1.602 \\
\hline 450 & 17 & 0.273 & -2.962 & -0.309 & 0.019 & 0.290 \\
\hline 480 & 18 & 0.476 & -1.369 & -0.904 & 0.001 & 0.903 \\
\hline \multicolumn{7}{|c|}{ Middle section } \\
\hline $\begin{array}{l}\text { Run } \\
\text { time }\end{array}$ & $\begin{array}{r}\mathbf{D E} \\
\mathbf{M}\end{array}$ & $\begin{array}{r}\text { Aggradation } \\
\left(A_{v f}\right)\end{array}$ & $\begin{array}{r}\text { Erosion } \\
\left(\boldsymbol{E}_{v f}\right)\end{array}$ & $\begin{array}{r}\text { Bank erosion } \\
\left(E_{b}\right)\end{array}$ & $\begin{array}{r}\text { Bank deposits } \\
\left(A_{b}\right)\end{array}$ & $\begin{array}{r}\text { Bank contribution } \\
\left(V_{b}\right)\end{array}$ \\
\hline 60 & 3 & 1.682 & -2.136 & -1.687 & 0.000 & 1.687 \\
\hline 90 & 4 & 1.411 & -1.829 & -3.157 & 0.000 & 3.157 \\
\hline 120 & 5 & 1.668 & -0.633 & -4.575 & 0.318 & 4.258 \\
\hline 150 & 6 & 1.588 & -0.769 & -5.283 & 0.000 & 5.283 \\
\hline 180 & 7 & 1.334 & -0.836 & -3.001 & 0.003 & 2.998 \\
\hline 210 & 8 & 0.352 & -2.967 & -2.863 & 0.017 & 2.845 \\
\hline 240 & 9 & 0.938 & -2.962 & -3.873 & 0.994 & 2.879 \\
\hline 270 & 11 & 0.791 & -1.751 & -2.602 & 0.015 & 2.586 \\
\hline 300 & 12 & 0.447 & -3.482 & -3.124 & 0.004 & 3.120 \\
\hline 330 & 13 & 0.678 & -2.051 & -5.551 & 0.010 & 5.541 \\
\hline 360 & 14 & 0.294 & -2.736 & -3.209 & 0.009 & 3.199 \\
\hline 390 & 15 & 0.712 & -3.250 & -1.085 & 0.690 & 0.395 \\
\hline 420 & 16 & 0.926 & -3.109 & -1.437 & 0.522 & 0.916 \\
\hline 450 & 17 & 0.837 & -3.403 & -1.709 & 0.026 & 1.682 \\
\hline 480 & 18 & 0.703 & -5.280 & -0.446 & 0.018 & 0.428 \\
\hline \multicolumn{7}{|c|}{ Lower section } \\
\hline $\begin{array}{l}\text { Run } \\
\text { time }\end{array}$ & $\begin{array}{r}\mathbf{D E} \\
\mathbf{M} \\
\end{array}$ & $\begin{array}{r}\text { Aggradation } \\
\left(A_{v f}\right) \\
\end{array}$ & $\begin{array}{r}\text { Erosion } \\
\left(E_{v f}\right) \\
\end{array}$ & $\begin{array}{r}\text { Bank erosion } \\
\left(E_{b}\right) \\
\end{array}$ & $\begin{array}{r}\text { Bank deposits } \\
\left(A_{b}\right) \\
\end{array}$ & $\begin{array}{r}\text { Bank contribution } \\
\left(V_{b}\right)\end{array}$ \\
\hline 60 & 3 & 0.116 & -3.941 & -4.646 & 0.000 & 4.646 \\
\hline 90 & 4 & 0.099 & -1.925 & -3.218 & 0.000 & 3.218 \\
\hline 120 & 5 & 0.397 & -1.817 & -2.224 & 0.003 & 2.221 \\
\hline 150 & 6 & 0.381 & -1.399 & -2.896 & 0.090 & 2.806 \\
\hline 180 & 7 & 1.269 & -1.001 & -5.157 & 0.776 & 4.382 \\
\hline 210 & 8 & 3.897 & -0.789 & -12.035 & 0.087 & 11.947 \\
\hline 240 & 9 & 0.773 & -2.528 & -10.169 & 0.044 & 10.124 \\
\hline 270 & 11 & 0.285 & -4.738 & -3.719 & 0.026 & 3.693 \\
\hline 300 & 12 & 0.605 & -3.440 & -3.396 & 0.112 & 3.283 \\
\hline 330 & 13 & 0.329 & -3.524 & -2.842 & 0.029 & 2.813 \\
\hline 360 & 14 & 0.548 & -2.966 & -3.685 & 0.015 & 3.669 \\
\hline 390 & 15 & 2.586 & -1.425 & -8.784 & 2.222 & 6.562 \\
\hline 420 & 16 & 0.837 & -3.309 & -8.083 & 0.023 & 8.060 \\
\hline 450 & 17 & 2.327 & -1.713 & -2.948 & 0.636 & 2.312 \\
\hline 480 & 18 & 1.212 & -3.872 & -8.081 & 0.217 & 7.864 \\
\hline
\end{tabular}

\section{Whole system}

\begin{tabular}{|c|c|c|c|c|c|c|c|}
\hline \multirow{2}{*}{ Run time } & \multirow{2}{*}{ DEM } & \multirow{2}{*}{ Aggradation $\left(A_{v f}\right)$} & \multirow{2}{*}{ Erosion $\left(E_{v f}\right)$} & \multirow{2}{*}{ Bank erosion $\left(E_{b}\right)$} & \multirow{2}{*}{ Bank deposits $\left(A_{b}\right)$} & \multicolumn{2}{|c|}{ Bank contribution } \\
\hline & & & & & & $\left(V_{b}\right)$ & $(\%)$ \\
\hline 60 & 3 & 3.542 & -6.894 & -6.816 & 0.000 & 6.816 & 67.0 \\
\hline 90 & 4 & 3.156 & -4.456 & -6.614 & 0.000 & 6.614 & 83.6 \\
\hline 120 & 5 & 3.470 & -3.062 & -6.863 & 0.320 & 6.543 & 106.7 \\
\hline 150 & 6 & 3.451 & -2.669 & -8.429 & 0.091 & 8.338 & 110.3 \\
\hline 180 & 7 & 4.123 & -2.324 & -8.272 & 0.779 & 7.493 & 131.6 \\
\hline 210 & 8 & 4.942 & -6.149 & -16.173 & 0.106 & 16.067 & 93.0 \\
\hline 240 & 9 & 1.923 & -8.787 & -15.008 & 1.043 & 13.965 & 67.0 \\
\hline
\end{tabular}




\begin{tabular}{|r|r|r|r|r|r|r|r|}
\hline 270 & 11 & 1.458 & -8.211 & -7.479 & 0.113 & 7.366 & 52.2 \\
\hline 300 & 12 & 1.170 & -9.939 & -7.008 & 0.118 & 6.890 & 44.0 \\
\hline 330 & 13 & 1.364 & -6.912 & -8.515 & 0.041 & 8.474 & 60.4 \\
\hline 360 & 14 & 1.162 & -6.716 & -8.426 & 0.177 & 8.249 & 59.8 \\
\hline 390 & 15 & 3.899 & -5.642 & -12.723 & 2.919 & 9.805 & 84.9 \\
\hline 420 & 16 & 2.097 & -7.379 & -11.129 & 0.548 & 10.581 & 66.7 \\
\hline 450 & 17 & 3.517 & -8.309 & -4.981 & 0.683 & 4.297 & 47.3 \\
\hline 480 & 18 & 2.482 & -10.735 & -9.785 & 0.238 & 9.547 & 53.6 \\
\hline
\end{tabular}

Table S9. Fan (a)symmetry measured from the DEMs.

\begin{tabular}{|c|c|c|c|c|}
\hline & Runtime & $\begin{array}{l}\text { Upstream } \\
\text { side }\end{array}$ & $\begin{array}{c}\text { Central } \\
\text { line }\end{array}$ & $\begin{array}{c}\text { Downstream } \\
\text { side }\end{array}$ \\
\hline & $(\min )$ & \multicolumn{3}{|c|}{ (m) } \\
\hline \multirow{9}{*}{$\begin{array}{l}\bar{U} \\
Z_{1}\end{array}$} & 120 & 0.665 & 0.745 & 0.898 \\
\hline & 180 & 0.549 & 0.699 & 0.884 \\
\hline & 240 & 0.595 & 0.638 & 0.78 \\
\hline & 300 & 0.607 & 0.635 & 0.714 \\
\hline & 360 & 0.531 & 0.664 & 0.741 \\
\hline & 420 & 0.523 & 0.626 & 0.706 \\
\hline & 480 & 0.512 & 0.745 & 0.946 \\
\hline & 540 & 0.621 & 0.645 & 0.814 \\
\hline & 600 & 0.63 & 0.741 & 0.905 \\
\hline \multirow{9}{*}{$\stackrel{\mathscr{2}}{\mathscr{2}}$} & 120 & 0.816 & 1.096 & 1.199 \\
\hline & 180 & 0.874 & 1.081 & 1.178 \\
\hline & 240 & 0.801 & 0.886 & 0.953 \\
\hline & 300 & 0.689 & 0.841 & 0.951 \\
\hline & 360 & 0.866 & 0.978 & 1.031 \\
\hline & 420 & 0.844 & 1.071 & 1.244 \\
\hline & 480 & 1.046 & 1.079 & 1.181 \\
\hline & 540 & 0.996 & 1.075 & 1.286 \\
\hline & 600 & 0.856 & 1.193 & 1.354 \\
\hline \multirow{9}{*}{$\sum_{i}^{\infty}$} & 120 & 0.829 & 0.994 & 1.181 \\
\hline & 180 & 0.845 & 0.887 & 0.935 \\
\hline & 240 & 0.698 & 0.799 & 0.896 \\
\hline & 300 & 0.697 & 0.832 & 0.948 \\
\hline & 360 & 0.804 & 0.949 & 1.066 \\
\hline & 420 & 0.772 & 0.869 & 1.199 \\
\hline & 480 & 0.82 & 1.067 & 1.211 \\
\hline & 540 & 0.859 & 1.111 & 1.032 \\
\hline & 600 & 0.778 & 1.252 & 1.356 \\
\hline \multirow{7}{*}{$\begin{array}{l}\bigcup_{Z_{1}} \\
Z_{1}\end{array}$} & 120 & 0.898 & 0.956 & 1.023 \\
\hline & 180 & 0.841 & 0.972 & 1.101 \\
\hline & 240 & 0.724 & 0.982 & 1.057 \\
\hline & 300 & 0.823 & 1.068 & 1.141 \\
\hline & 360 & 0.768 & 0.889 & 1.016 \\
\hline & 420 & 0.784 & 1.077 & 1.156 \\
\hline & 480 & 0.7 & 0.942 & 1.122 \\
\hline \multirow{7}{*}{$\sum_{\underbrace{\prime}_{1}}^{U}$} & 120 & 0.848 & 0.961 & 1.023 \\
\hline & 180 & 0.803 & 0.891 & 1.031 \\
\hline & 240 & 0.801 & 0.862 & 0.997 \\
\hline & 300 & 0.842 & 0.977 & 1.071 \\
\hline & 360 & 0.741 & 0.811 & 0.996 \\
\hline & 420 & 0.788 & 0.940 & 1.055 \\
\hline & 480 & 0.692 & 0.796 & 1.08 \\
\hline
\end{tabular}



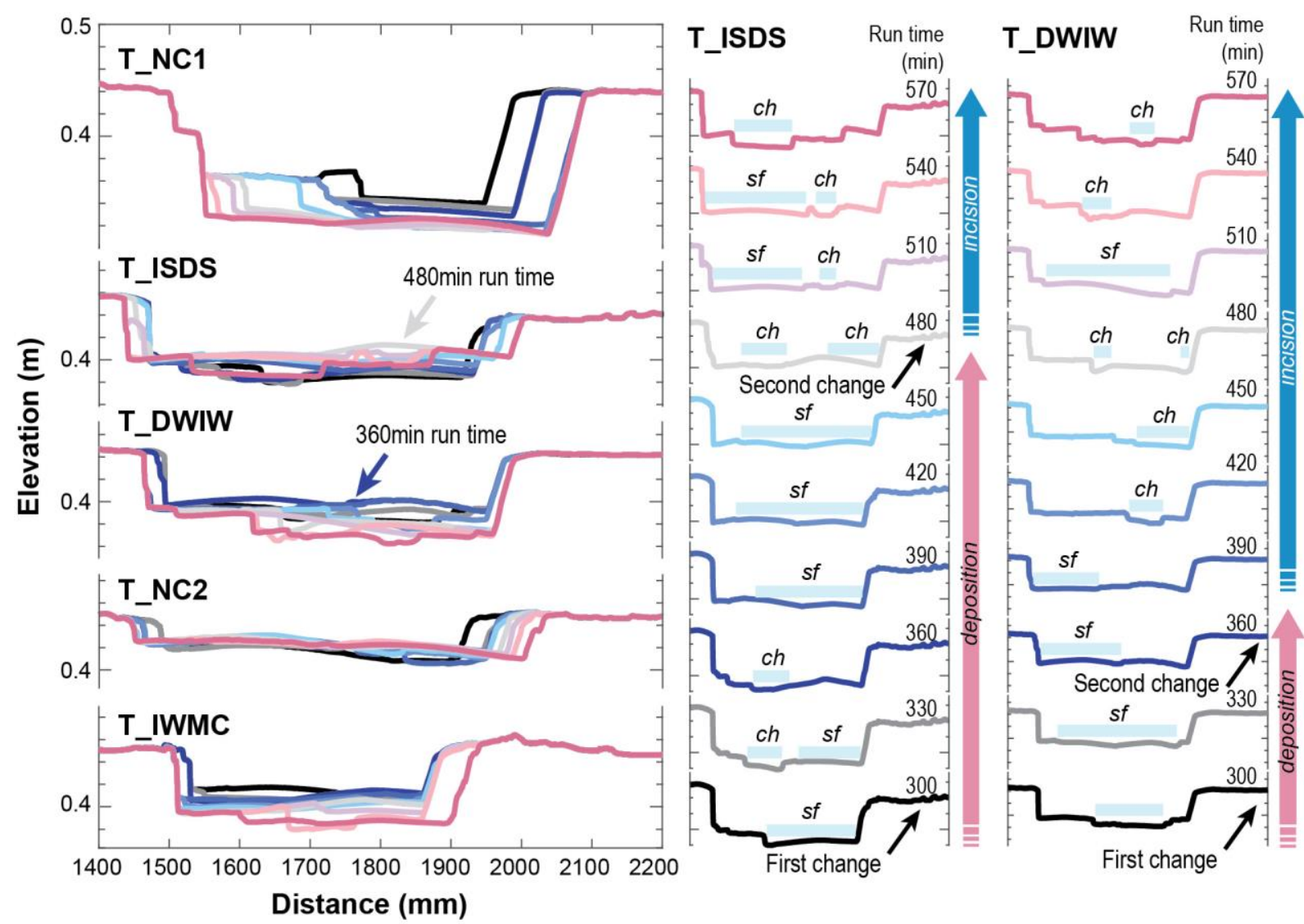

Figure S1. Cross sections in the tributary drawn from left to right looking downstream. The left panels show the evolution of all runs (color code as in Fig. 4 and 5); the right panels show the evolution of the T_ISDS and T_DWIW runs in more detail: the ground-surface elevation (colored lines) and the wetted areas (light blue bars) are shown. During aggradation, sheet flows ( $s f$ ) dominate the transport mode of sediment, although channels (ch) may contemporaneously be present on the fan surface. During incision, the flow alternates between channelized flows and sheet flows and contribute to lowering the entire fan topography. 


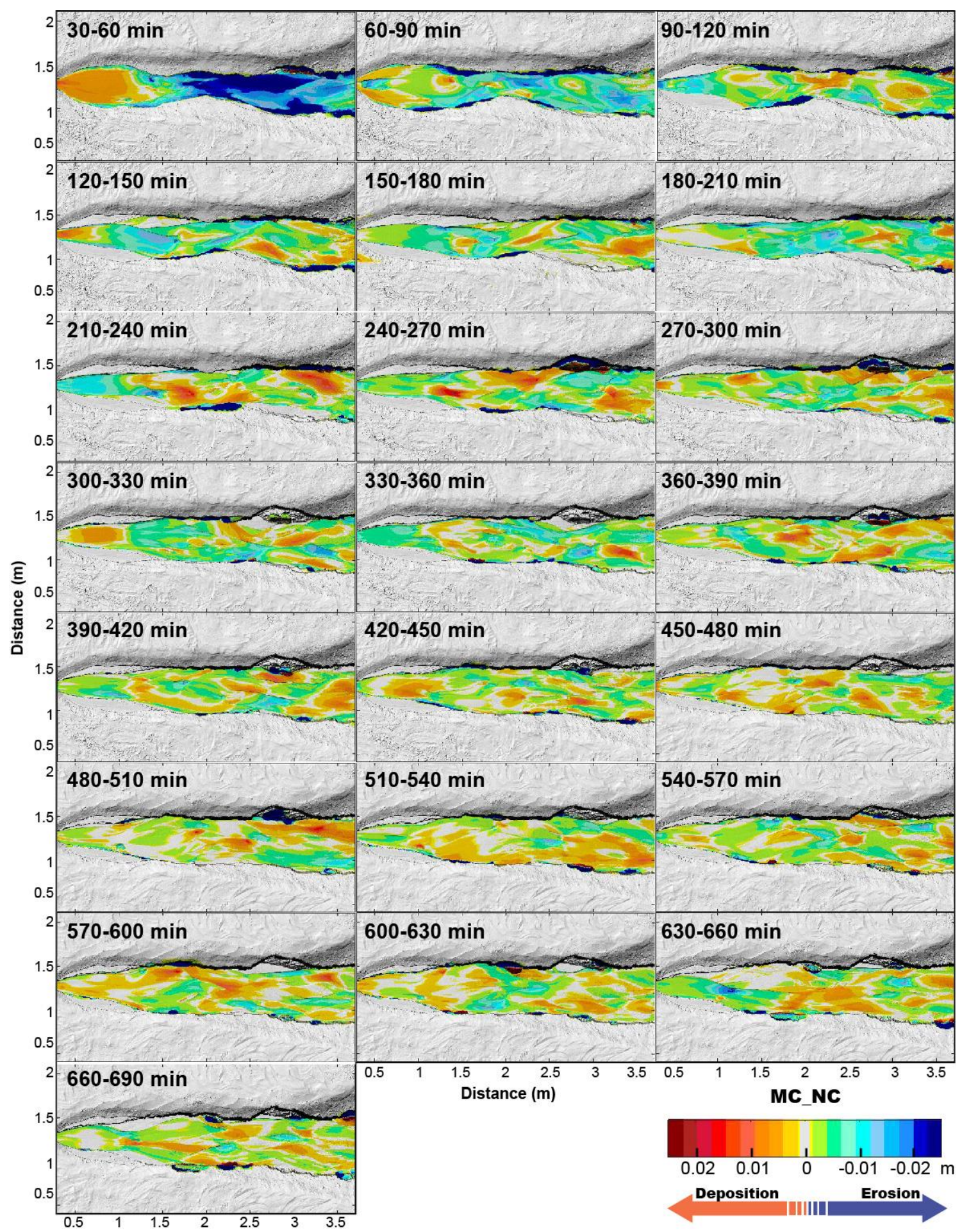

Figure S2. Sediment transfer dynamics from DoDs analysis. Variations between -0.001 and $+0.001 \mathrm{~m}$ are considered as "no change" (in gray) to account for the DEMs accuracy (i.e., $1 \mathrm{~mm}$ resolution). 


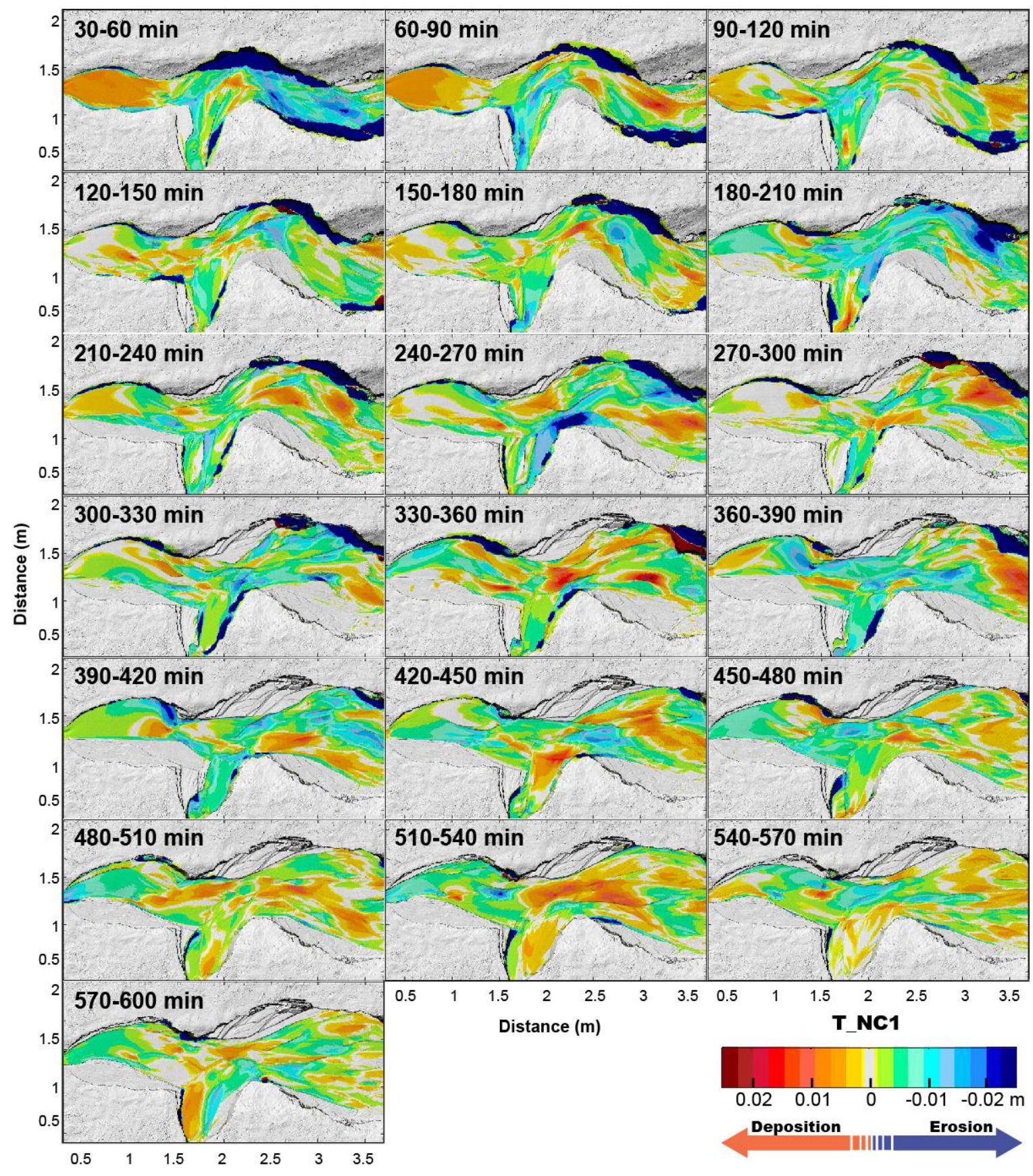

Figure S3. Sediment transfer dynamics from DoDs analysis. Variations between -0.001 and $+0.001 \mathrm{~m}$ are considered as "no change" (in gray) to account for the DEMs accuracy (i.e., $1 \mathrm{~mm}$ resolution). 
(a) $Q_{s_{-} \text {in (TRIBUTARY) }}=2.2 \mathrm{~mL} / \mathrm{s}$

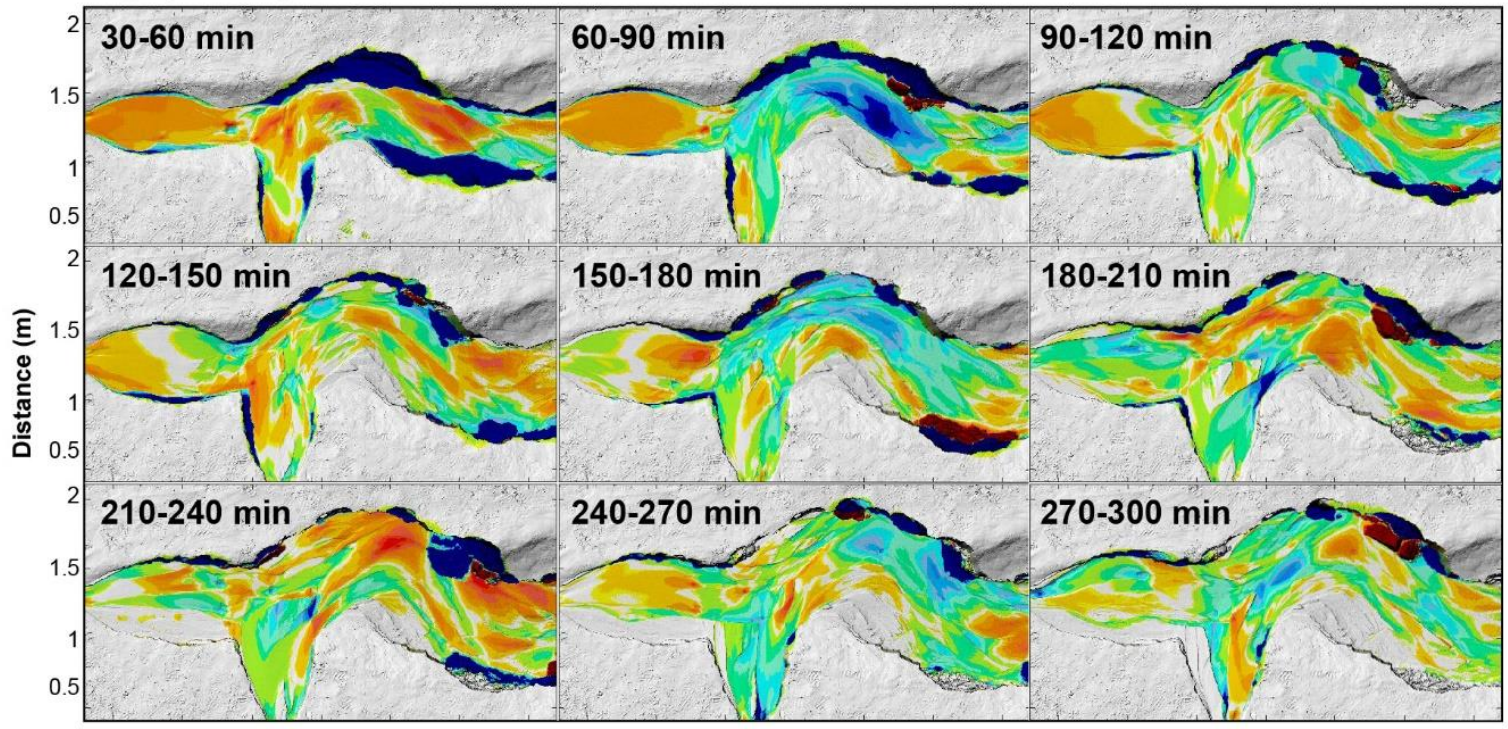

(b) Increasing sediment $\left(Q_{s \text { in (TRIBUTARY) }}=4.5 \mathrm{~mL} / \mathrm{s}\right)$

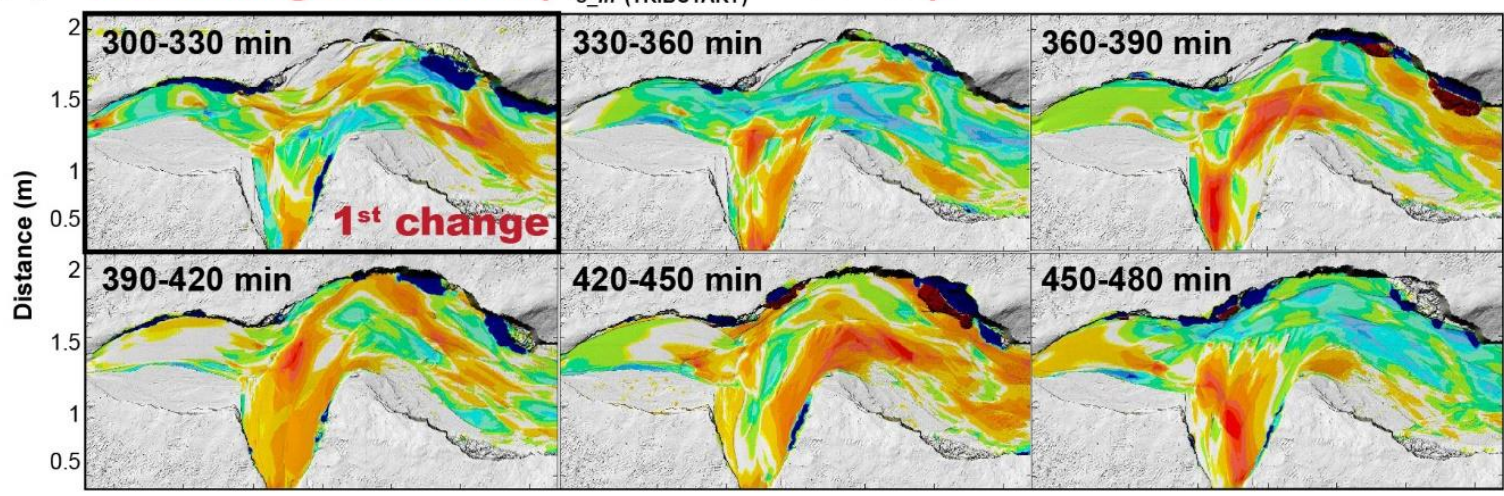

(c) Decreasing sediment $\left(Q_{s_{-} \text {in (TRIBUTARY) }}=\mathbf{2 . 2} \mathrm{mL} / \mathrm{s}\right)$

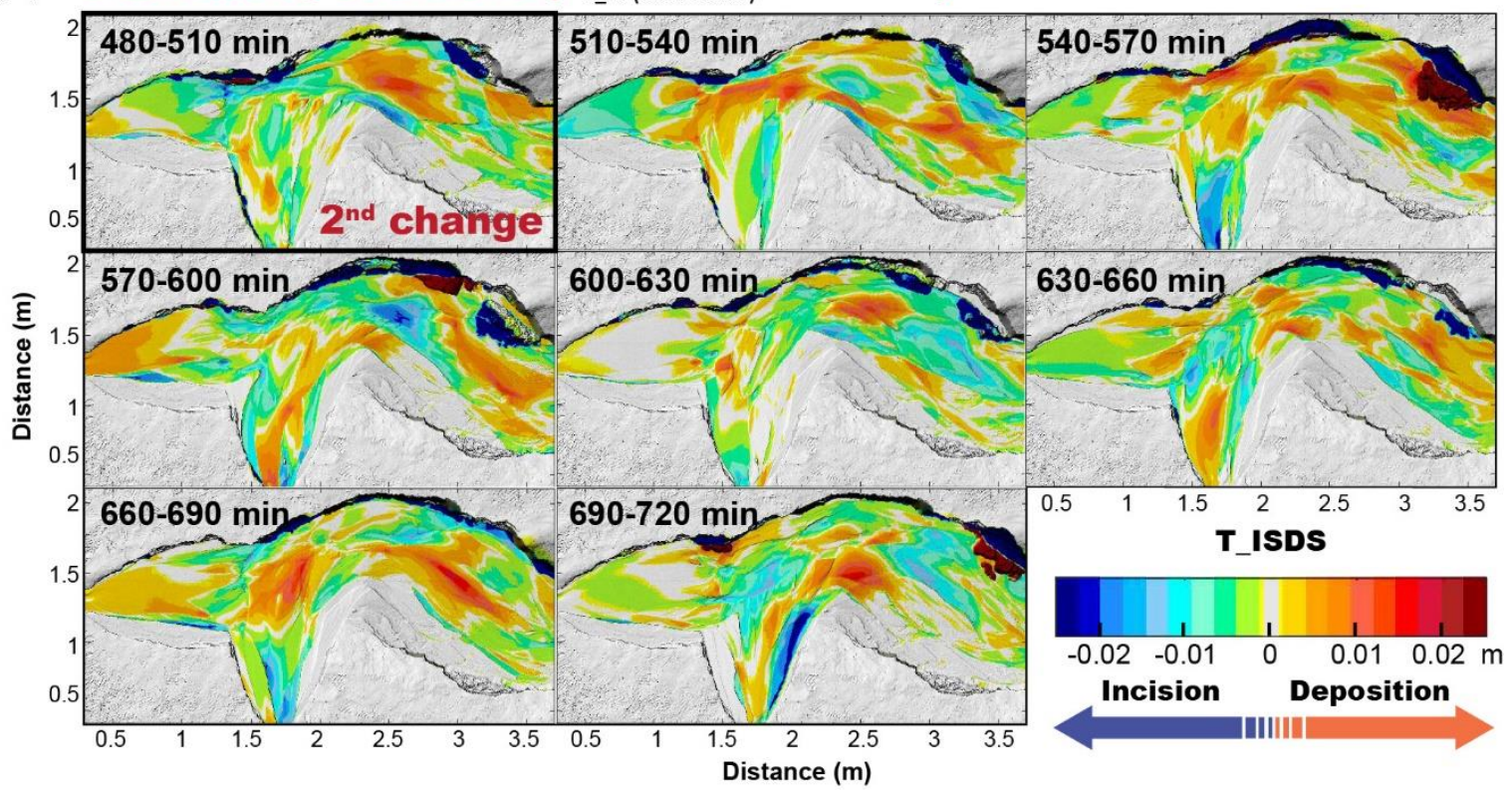


Figure S4. Sediment transfer dynamics within the system in the T_ISDS experiment (from DoDs analysis). Variations between -0.001 and $+0.001 \mathrm{~m}$ are considered as "no change" (in gray) to account for the DEMs accuracy (i.e., $1 \mathrm{~mm}$ resolution). (a) Pre-perturbation phase (between 30 and 150 minutes is considered to be the spin-up phase); (b) Fan aggradation (300-390 min) and progradation (390-480 min) phase; (c) Fan incision and progradation phase (480 min until end of run). 

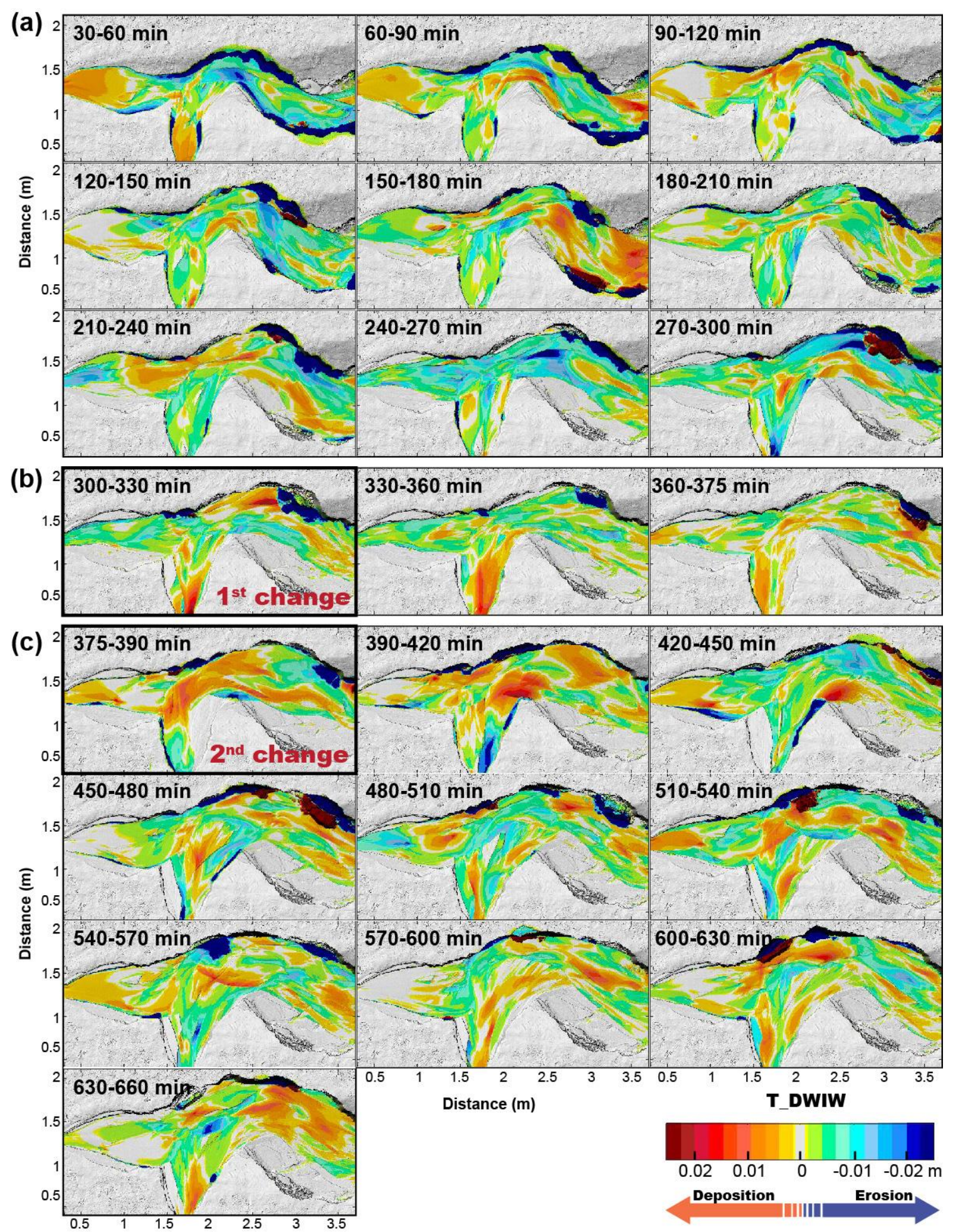

Figure S5. Sediment transfer dynamics from DoDs analysis. Variations between -0.001 and $+0.001 \mathrm{~m}$ are considered as "no change" (in gray) to account for the DEMs accuracy (i.e., $1 \mathrm{~mm}$ resolution). 


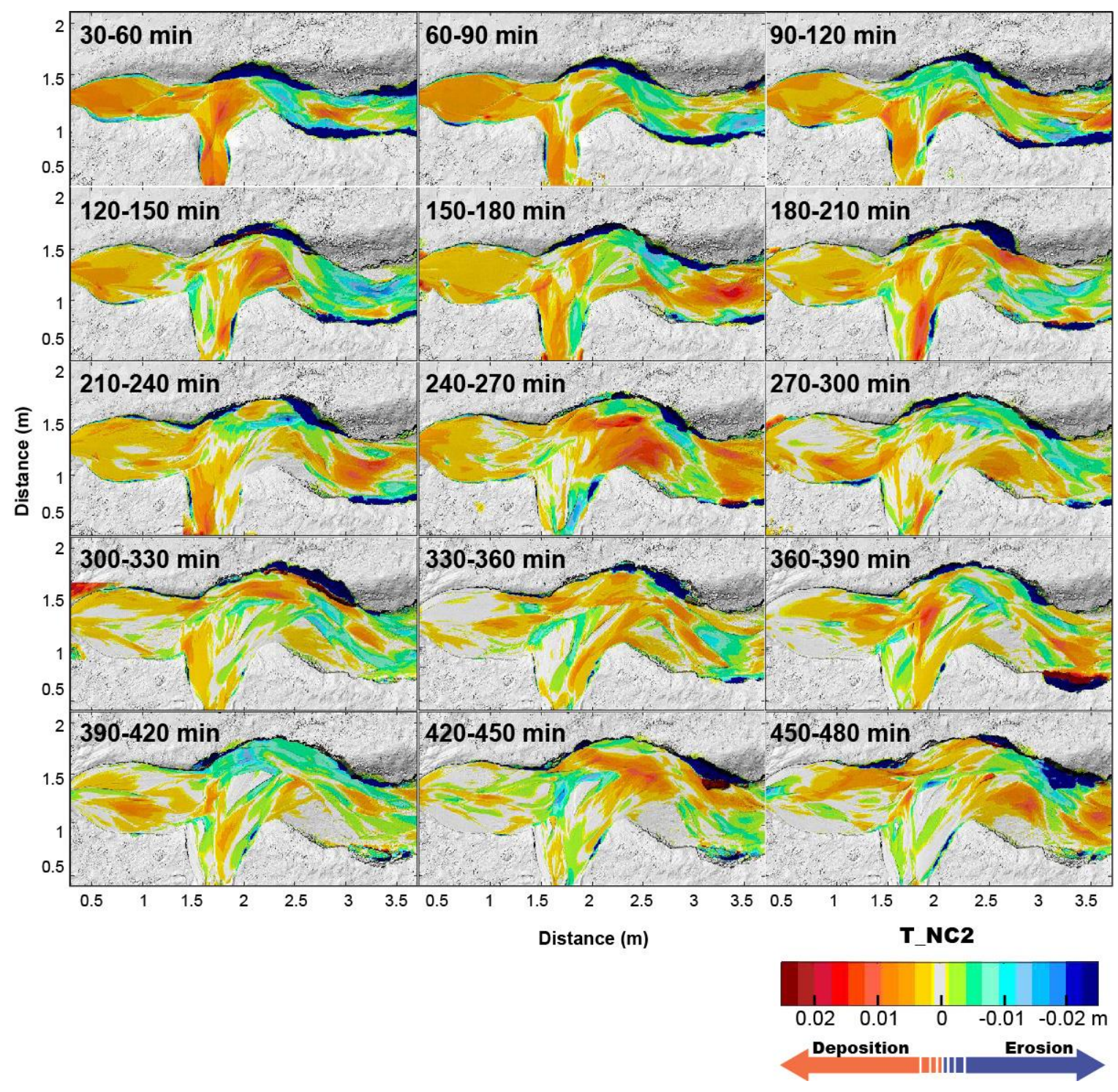

Figure S6. Sediment transfer dynamics from DoDs analysis. Variations between -0.001 and $+0.001 \mathrm{~m}$ are considered as "no change" (in gray) to account for the DEMs accuracy (i.e., $1 \mathrm{~mm}$ resolution). 

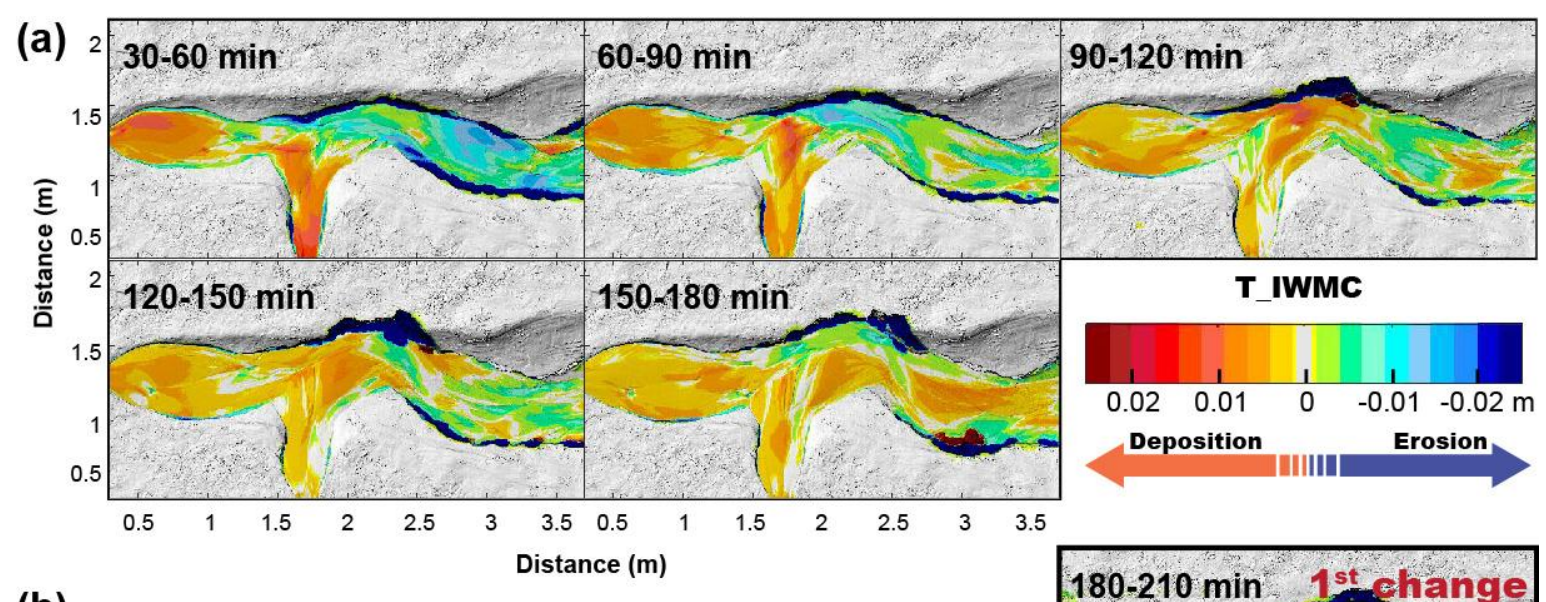

(b)
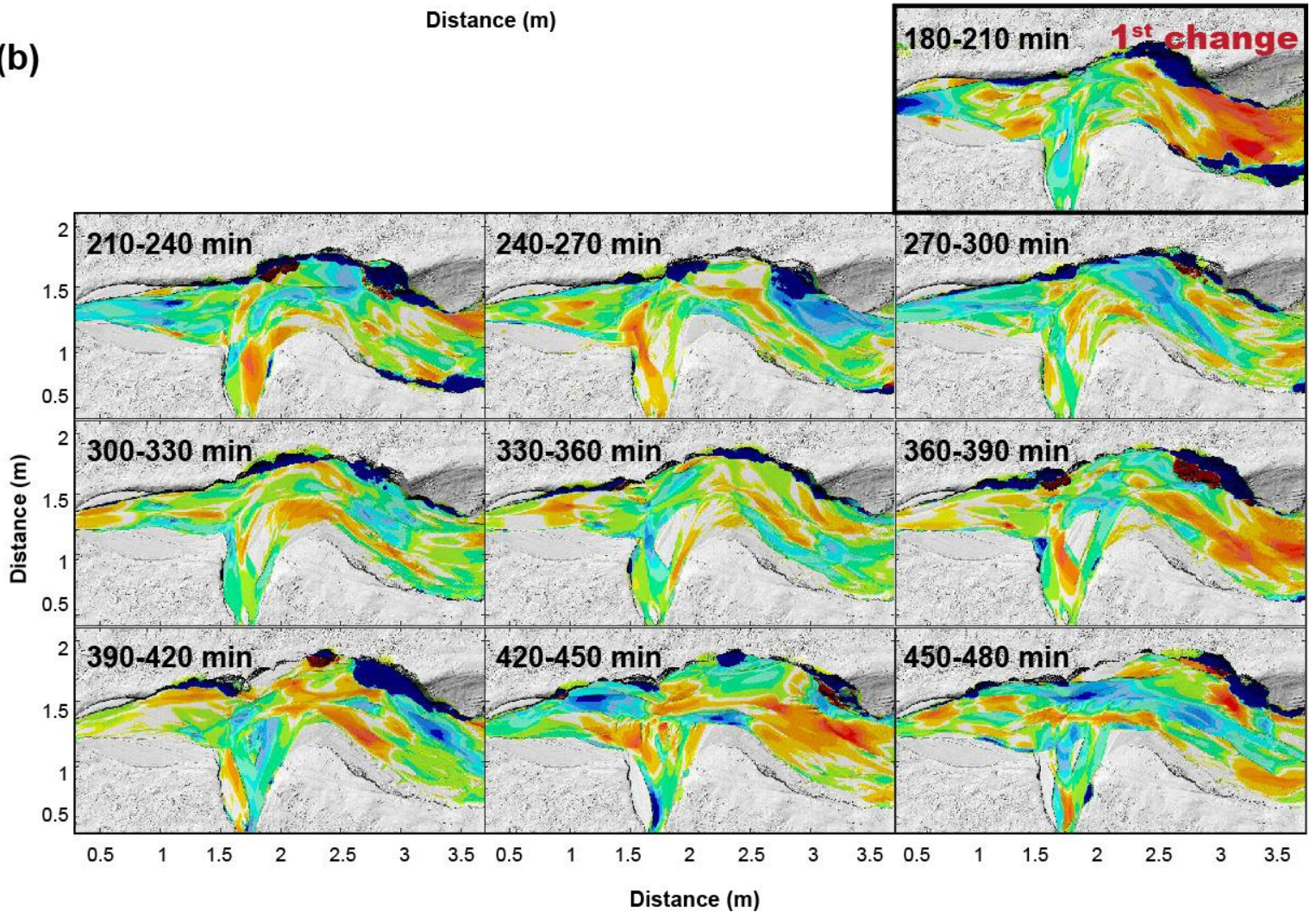

Figure S7. Sediment transfer dynamics from DoDs analysis. Variations between -0.001 and $+0.001 \mathrm{~m}$ are considered as "no change" (in gray) to account for the DEMs accuracy (i.e., $1 \mathrm{~mm}$ resolution). 


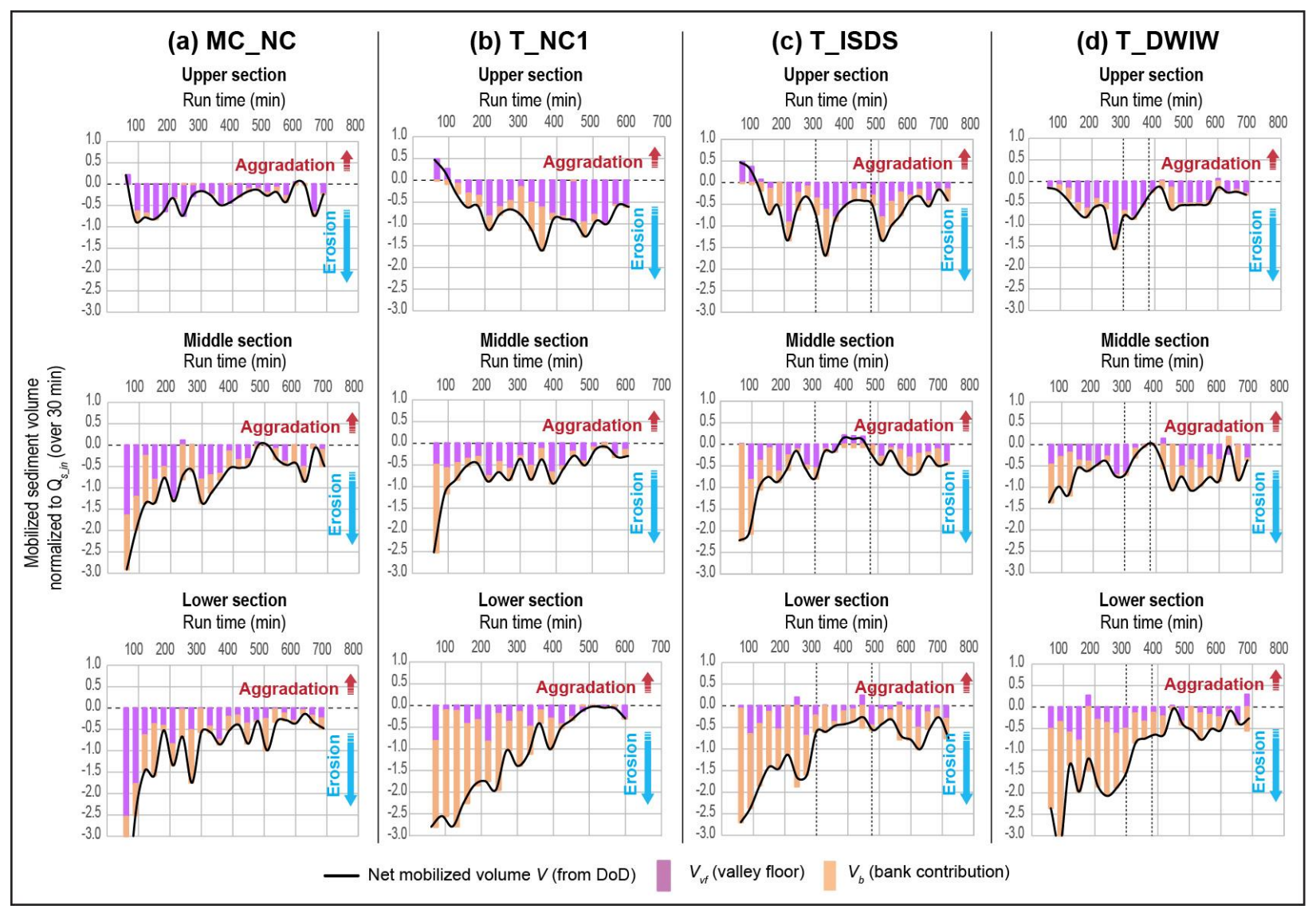

Figure S8. Net volume of sediment mobilized in the upper, middle, and lower sections (normalized to $Q_{s_{-} i n}$ ). Net $V$ values are in black; bank contribution $\left(V_{b}\right)$ is in orange, whereas valley floor $\left(V_{a f}\right)$ is in violet. 


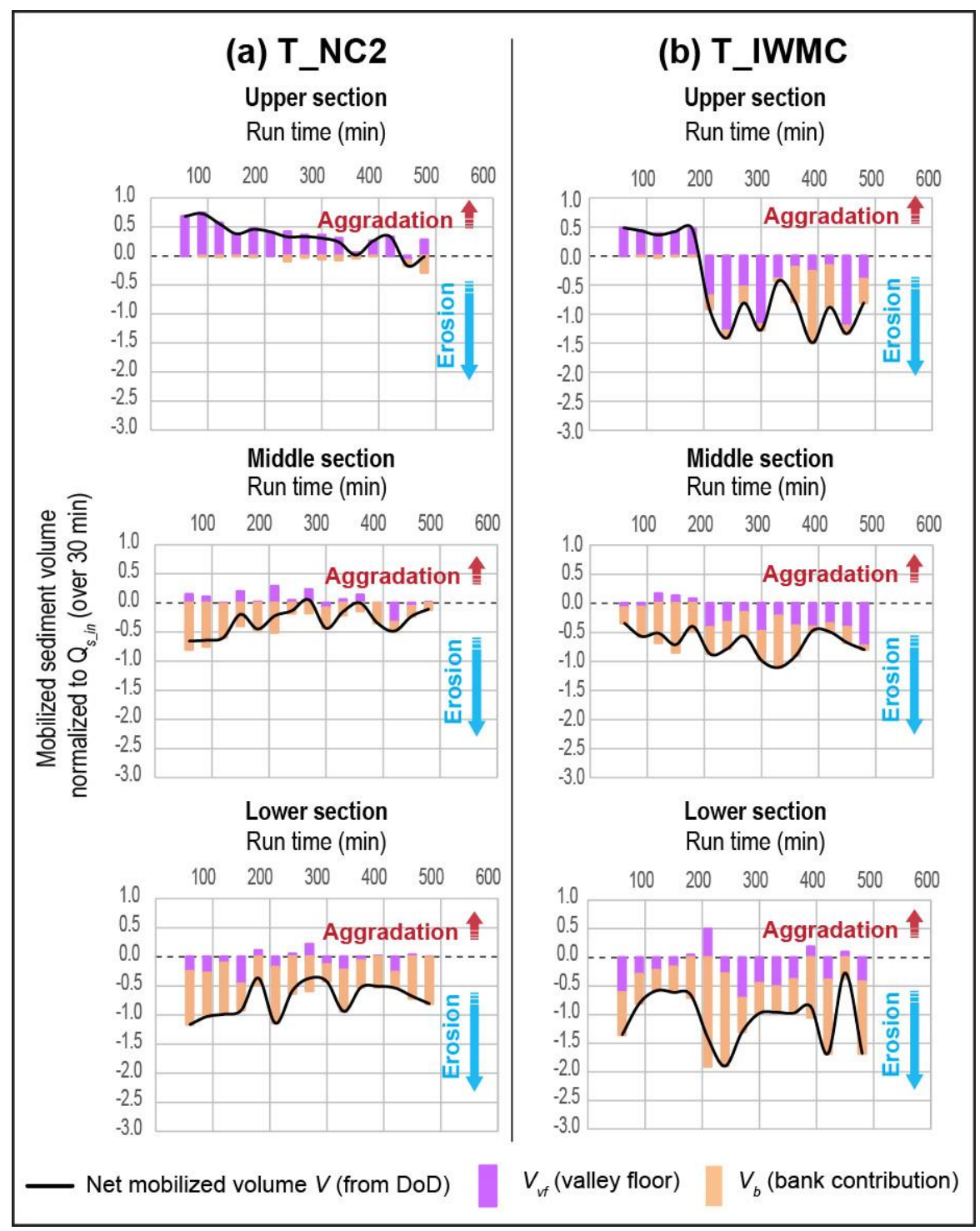

Figure S9. Net volume of sediment mobilized in the upper, middle, and lower sections (normalized to $Q_{s_{-} i n}$ ). Net $V$ values are in black; bank contribution $\left(V_{b}\right)$ is in orange, whereas valley floor $\left(V_{a f}\right)$ is in violet. 